\title{
Effects of (Multi)branching of Dipolar Chromophores on Photophysical Properties and Two-Photon Absorption
}

\author{
Claudine Katan, ${ }^{\dagger}$ Francesca Terenziani, ${ }^{\dagger}$ Olivier Mongin, ${ }^{\dagger}$ Martinus H. V. Werts,${ }^{\dagger}$ \\ Laurent Porrès, ${ }^{\dagger}$ Thomas Pons, ${ }^{\dagger}$ Jerome Mertz, ${ }^{\ddagger}, \S$ Sergei Tretiak, ${ }^{*, l l}$ and \\ Mireille Blanchard-Desce*, ${ }^{*}$
}

Synthèse et ElectroSynthèse Organiques (CNRS, UMR 6510), Université de Rennes 1, Institut de Chimie, Campus Scientifique de Beaulieu, Bât 10A, F-35042 Rennes Cedex, France, Neurophysiologie et Nouvelles Microscopies (INSERM EPI 00-02, CNRS FRE 2500), Ecole Supérieure de Physique et Chimie Industrielles, 10 rue Vauquelin, F-75231 Paris Cedex 05, France, and Los Alamos National Laboratory, Theoretical Division, Los Alamos, New Mexico 87545

Received: December 21, 2004; In Final Form: February 1, 2005

\begin{abstract}
To investigate the effect of branching on linear and nonlinear optical properties, a specific series of chromophores, epitome of (multi)branched dipoles, has been thoroughly explored by a combined theoretical and experimental approach. Excited-state structure calculations based on quantum-chemical techniques (timedependent density functional theory) as well as a Frenkel exciton model nicely complement experimental photoluminescence and one- and two-photon absorption findings and contribute to their interpretation. This allowed us to get a deep insight into the nature of fundamental excited-state dynamics and the nonlinear optical (NLO) response involved. Both experiment and theory reveal that a multidimensional intramolecular charge transfer takes place from the donating moiety to the periphery of the branched molecules upon excitation, while fluorescence stems from an excited state localized on one of the dipolar branches. Branching is also observed to lead to cooperative enhancement of two-photon absorption (TPA) while maintaining high fluorescence quantum yield, thanks to localization of the emitting state. The comparison between results obtained in the Frenkel exciton scheme and ab initio results suggests the coherent coupling between branches as one of the possible mechanisms for the observed enhancement. New strategies for the rational design of NLO molecular assemblies are thus inferred on the basis of the acquired insights.
\end{abstract}

\section{Introduction}

Organic molecular materials with high nonlinear optical (NLO) responses have diverse and important technical issues. In the quest of improving NLO properties, attention has been essentially focused on dipolar molecules for several decades. ${ }^{1-3}$ More recently, quadrupolar systems have been designed and investigated $^{4-21}$ and have shown improved properties, in particular with respect to the trade-off between optical transparency and NLO performance. Lately, attention has turned toward multipolar ${ }^{22-33}$ and branched structures including dendrimers. ${ }^{23,24,28,33-43}$ Given the huge synthetic effort needed to build such supramolecular structures, a detailed understanding of the effect of branching of molecular entities on the linear and nonlinear optical properties is of major interest. Thus, further development of approaches for rational design of NLO assemblies is needed in order to focus the synthesis on knowledgebased materials. Among the many questions that are still debated, coherent coupling between the arms (building blocks) is of particular interest. Depending on the nature of this coupling, the ground state or the excited state may be either localized or

* E-mail: mireille.blanchard-desce@univ-rennes1.fr (M.B.-D.); serg@ lanl.gov (S.T.).

Université de Rennes 1.

$\div$ Ecole Supérieure de Physique et Chimie Industrielles.

$\S$ Present address: Boston University, BME Dept., 44 Cummington St. Boston, MA 02215 .

"Los Alamos National Laboratory. delocalized, and specific optical properties may be either enhanced or suppressed. ${ }^{44}$

Among the many NLO effects, two-photon absorption (TPA) has become very popular during the past decade owing to its wide-ranging applications such as two-photon laser scanning microscopy, ${ }^{45-47}$ photodynamic therapy, ${ }^{48}$ optical power limitation, ${ }^{4,5}$ microfabrication, ${ }^{49-52}$ or $3 \mathrm{D}$ optical data storage. ${ }^{53-56}$ Depending on the applications, two-photon chromophores have to satisfy different kinds of requirements. For instance, the combination of high fluorescence quantum yield $(\phi)$ and TPA cross-section $\left(\sigma_{2}\right)$ in the red-NIR range $(700-1200 \mathrm{~nm})$ is desirable for biological imaging. A number of factors influence the TPA magnitude, among which are electronic delocalization and intramolecular charge-transfer phenomena. In recent years, considerable effort has been devoted to the design and investigation of chromophores with large TPA cross-sections, exploring in particular the possibility of obtaining optimized response due to the multipolar and/or (multi)branching strategy. ${ }^{57}$ The experimental investigation of the branching effect has led to various trends: cooperative enhancement, ${ }^{24,38,40,41}$ additive behavior, ${ }^{36,37}$ or even reduction of TPA $^{37}$ have been reported. Among the reasons that lead to these apparently conflicting findings, two can be put forward. First, most of these studies were limited to single-wavelength measurements, ${ }^{24,25,31,35-37,40}$ which can obviously lead to discrepancies, because spectral position and shape always play an important role. Second, the nature of the branches (dipolar versus quadrupolar, for example) 


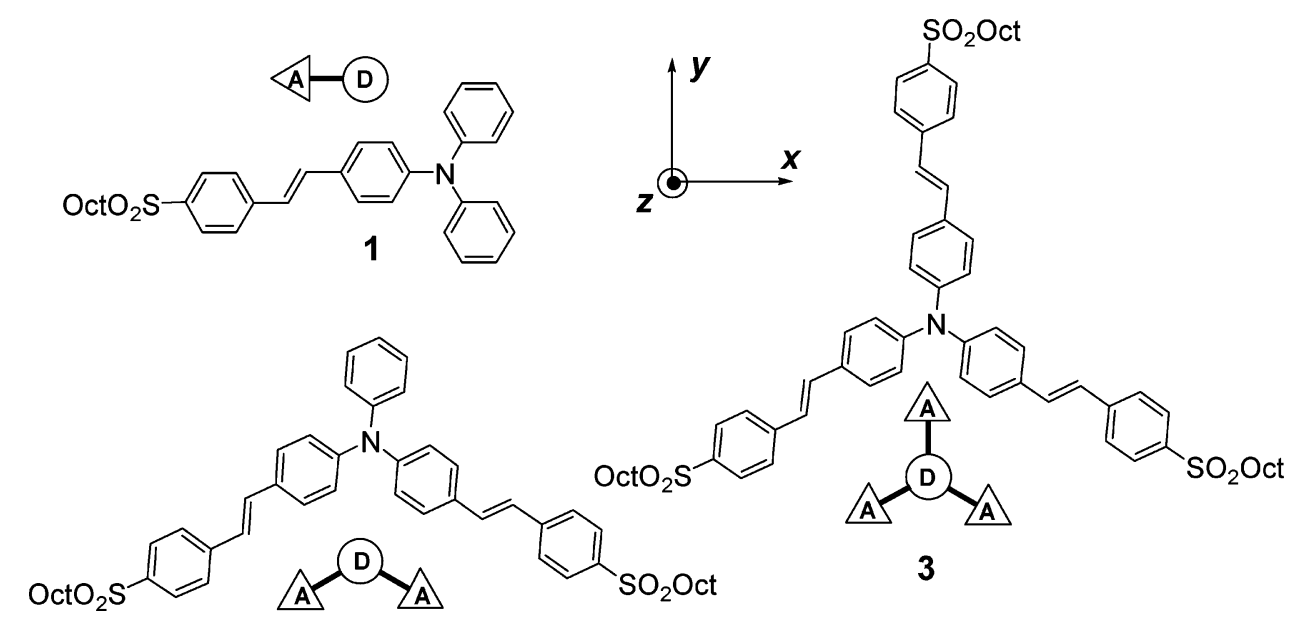

Figure 1. Series of structurally related dipolar (1), V-shaped (2), and octupolar (3) analogues derived from the functionalization of a triphenylamine moiety.

will also be of major importance, as it affects interbranch coupling.

The aim of this paper is to thoroughly investigate the effect of branching of dipolar chromophores on photoluminescence and TPA by combining various experimental and theoretical approaches. These include UV-vis spectra, fluorescence, fluorescence decay, and femtosecond two-photon-excited fluorescence (TPEF) measurements, high-level (state-of-the-art) quantum-chemical approaches as well as simple interpretative models. The paper will focus on the branching of dipolar entities in the weak-medium interaction limit. An asymmetrically substituted stilbene chromophore bearing an electron-withdrawing end group ( $\mathrm{SO}_{2} \mathrm{Oct}$, Figure 1$)$ has been chosen as a prototype for this class. The grafting of one, two, or three branches on an electron-donating trigonal moiety (triphenylamine) leads to multipolar chromophores of different symmetry: dipolar, Vshaped, and octupolar. Triphenylamine is a well-known electrondonating moiety, which has been used both in the design of octupoles for second-order NLO effects ${ }^{58-61}$ and in branched and dendritic structures for molecular TPA. ${ }^{23-25,29-32,36-38,41,42}$

In this paper, we first briefly describe the synthesis of the studied chromophores, as well as experimental and computational details (section 2). Linear absorption and fluorescence properties of the compounds are then thoroughly explored through a combined analysis of both experimental and theoretical results (section 3). In particular, the solvatochromic behavior of one-photon spectra, combined with excited-state lifetimes, suggests an emitting excited state localized on one branch. This phenomenon of localization is investigated and demonstrated through quantum-chemical excited-state calculations. Twophoton spectra of branched chromophores analyzed in section 4 show a cooperative enhancement with respect to the dipolar analogue in the visible red region as well as in the near-IR. These results can be qualitatively interpreted as resulting from the splitting of excited states due to the coupling between the dipolar branches. Theoretical modeling of TPA spectra with time-dependent density functional theory (TD-DFT) and the interpretation of interactions in the branched chromophores using a Frenkel exciton model allows us to confirm and explain all observed experimental trends. Finally, in section 5, we summarize the observed trends and suggest new routes for TPA enhancement and for the rational design of optimized structures.

\section{Experimental and Computational Details}

2.1. Synthesis of the Chromophores. Chromophores 1-3 were prepared in one step by means of a single, double, or triple
SCHEME 1: Synthesis of Chromophores 1-3

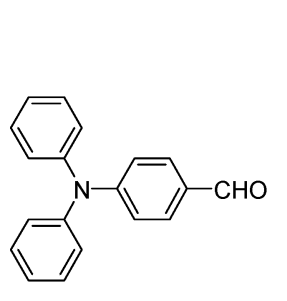

5
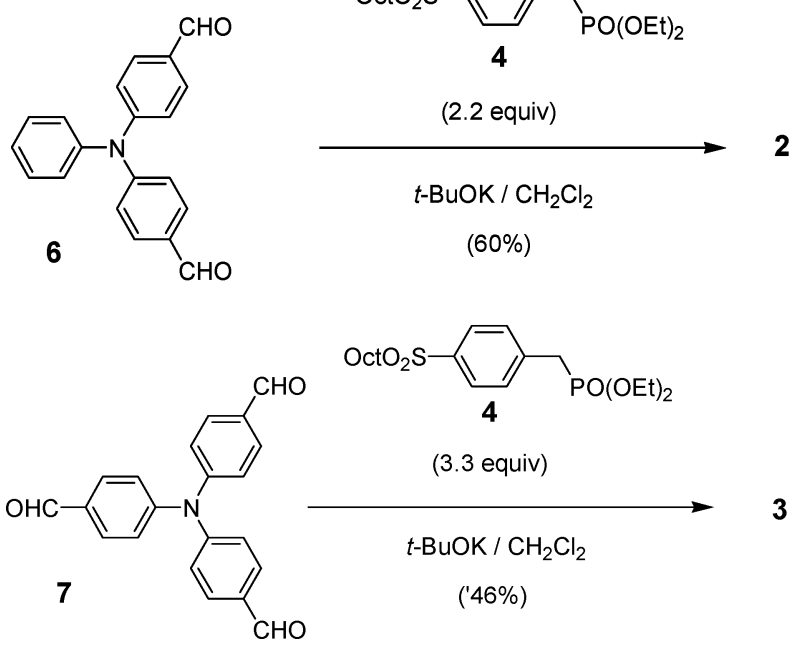

Horner-Wadsworth-Emmons condensation carried out in solid-liquid phase-transfer conditions by reacting either 1.1, 2.2 , or 3.3 equiv of phosphonate reagent 4 with either aldehyde $\mathbf{5}, \mathbf{6}$, or $\mathbf{7}^{62,63}$ (Scheme 1). Chromophores 1-3 were obtained as pure all- $E$ compounds as testified by ${ }^{1} \mathrm{H}$ NMR spectra. Thanks to the presence of the alkyl chains on the electron-withdrawing end groups, chromophores $\mathbf{1 - 3}$ maintain suitable solubility in chlorinated solvents.

4-[(1E)-2-[4-(Octylsulfonyl)phenyl]ethenyl]-N,N-diphenylbenzenamine (1). To a solution of 4-(diphenylamino)benzaldehyde $^{62}(\mathbf{5})(94.5 \mathrm{mg}, 0.346 \mathrm{mmol})$ and 4 (140 $\left.\mathrm{mg}, 0.346 \mathrm{mmol}\right)$ in anhyd $\mathrm{CH}_{2} \mathrm{Cl}_{2}(8 \mathrm{~mL})$ was added $t$-BuOK $(58 \mathrm{mg}, 0.52$ mmol). The mixture was stirred at $20{ }^{\circ} \mathrm{C}$ for $16 \mathrm{~h}$, and the solvent was removed under reduced pressure. After the addition of water, extraction with $\mathrm{CH}_{2} \mathrm{Cl}_{2}$, and drying $\left(\mathrm{Na}_{2} \mathrm{SO}_{4}\right)$, the solvent was evaporated. The crude product was purified by column chromatography (heptane/ $\mathrm{CH}_{2} \mathrm{Cl}_{2}, 60: 40$ ) to afford a 
mixture of isomers, which was dissolved in $\mathrm{Et}_{2} \mathrm{O}(25 \mathrm{~mL})$. A catalytic amount of $\mathrm{I}_{2}(0.3 \%)$ was then added, and the solution was stirred at $20{ }^{\circ} \mathrm{C}$ for $2 \mathrm{~h}$ under light exposure (75-W lamp). The organic layer was washed with aq $\mathrm{Na}_{2} \mathrm{~S}_{2} \mathrm{O}_{3}$ and dried $\left(\mathrm{Na}_{2}-\right.$ $\mathrm{SO}_{4}$ ). After evaporation of the solvent, the crude product was purified by filtration through a pad of silica gel (heptane/ $\mathrm{CH}_{2}$ $\left.\mathrm{Cl}_{2}, 65: 35\right)$ to yield $97 \mathrm{mg}(54 \%)$ of 1 : $\mathrm{mp} 79{ }^{\circ} \mathrm{C} ;{ }^{1} \mathrm{H} \mathrm{NMR}$ $\left(200.13 \mathrm{MHz}, \mathrm{CDCl}_{3}\right) \delta 7.85$ and $7.63\left(\mathrm{AA}^{\prime} \mathrm{XX}^{\prime}, J_{\mathrm{AX}}=8.5\right.$, $4 \mathrm{H}), 7.40$ and $7.08\left(\mathrm{AA}^{\prime} X^{\prime}, J_{\mathrm{AX}}=8.6,4 \mathrm{H}\right), 7.28(\mathrm{~m}, 4 \mathrm{H})$, $7.20(\mathrm{~d}, J=16.6,1 \mathrm{H}), 7.11(\mathrm{~m}, 6 \mathrm{H}), 6.99(\mathrm{~d}, J=16.6,1 \mathrm{H})$, $3.08(\mathrm{~m}, 2 \mathrm{H}), 1.72(\mathrm{~m}, 2 \mathrm{H}), 1.24(\mathrm{~m}, 10 \mathrm{H}), 0.86(\mathrm{t}, J=6.5$, $3 \mathrm{H}) ;{ }^{13} \mathrm{C} \mathrm{NMR}\left(50.32 \mathrm{MHz}, \mathrm{CDCl}_{3}\right) \delta 148.3,147.2,143.0$, $146.8,132.0,129.9,129.3,128.4,127.8,126.6,124.8,124.5$, $123.4,122.8,56.4,31.6,28.9,28.8,28.2,22.7,22.5,14.0$; HRMS (LSIMS ${ }^{+}$, mNBA) calcd for $\mathrm{C}_{34} \mathrm{H}_{37} \mathrm{NO}_{2} \mathrm{~S}\left(\mathrm{M}^{+\bullet}\right) \mathrm{m} / \mathrm{z}$ 523.2545, found 523.2519.

Bis[4-[(1E)-2-[4-(octylsulfonyl)phenyl]ethenyl]phenyl]phenylamine (2). To a solution of bis(4-formylphenyl)phenylamine ${ }^{62}$ (6) (90.4 mg, $0.3 \mathrm{mmol})$ and $4(267 \mathrm{mg}, 0.66 \mathrm{mmol})$ in anhyd $\mathrm{CH}_{2} \mathrm{Cl}_{2}(8 \mathrm{~mL})$ was added $t$-BuOK $(100 \mathrm{mg}, 0.90 \mathrm{mmol})$. The mixture was stirred at $20{ }^{\circ} \mathrm{C}$ for $16 \mathrm{~h}$, and the solvent was removed under reduced pressure. After the addition of water, extraction with $\mathrm{CH}_{2} \mathrm{Cl}_{2}$, and drying $\left(\mathrm{Na}_{2} \mathrm{SO}_{4}\right)$, the solvent was evaporated. The crude product was purified by column chromatography (heptane/ $\mathrm{CH}_{2} \mathrm{Cl}_{2}, 20: 80$ ) to yield $144 \mathrm{mg}(60 \%)$ of 2: $\operatorname{mp} 68{ }^{\circ} \mathrm{C}$; ${ }^{1} \mathrm{H}$ NMR $\left(200.13 \mathrm{MHz}, \mathrm{CDCl}_{3}\right) \delta 7.86$ and 7.64 $\left(\mathrm{AA}^{\prime} \mathrm{XX}^{\prime}, J_{\mathrm{AX}}=8.4,8 \mathrm{H}\right), 7.44$ and $7.11\left(\mathrm{AA}^{\prime} \mathrm{XX}^{\prime}, J_{\mathrm{AX}}=8.7\right.$, $8 \mathrm{H}), 7.32(\mathrm{~m}, 2 \mathrm{H}), 7.22(\mathrm{~d}, J=16.7,2 \mathrm{H}), 7.15(\mathrm{~m}, 3 \mathrm{H}), 7.02$ $(\mathrm{d}, J=16.7,2 \mathrm{H}), 3.09(\mathrm{~m}, 4 \mathrm{H}), 1.72(\mathrm{~m}, 4 \mathrm{H}), 1.23(\mathrm{~m}, 20 \mathrm{H})$, $0.86(\mathrm{t}, J=6.5,6 \mathrm{H}) ;{ }^{13} \mathrm{C} \mathrm{NMR}\left(75.47 \mathrm{MHz}, \mathrm{CDCl}_{3}\right) \delta 147.7$, $146.8,142.9,137.0,131.9,130.8,129.5,128.5,127.9,126.7$, 125.3, 125.0, 124.1, 123.7, 56.4, 31.6, 28.95, 28.87, 28.3, 22.7, $22.5,14.0$; HRMS (LSIMS ${ }^{+}$, mNBA) calcd for $\mathrm{C}_{50} \mathrm{H}_{59} \mathrm{NO}_{4} \mathrm{~S}_{2}$ $\left(\mathrm{M}^{+\bullet}\right) \mathrm{m} / \mathrm{z}$ 801.3886, found 801.3869. Anal. Calcd for $\mathrm{C}_{50} \mathrm{H}_{59^{-}}$ $\mathrm{NO}_{4} \mathrm{~S}_{2}$ (802.16): C, 74.87; H, 7.41; N, 1.75. Found: C, 74.89; H, 7.39; N, 1.69.

Tris[4-[(1E)-2-[4-(octylsulfonyl)phenyl]ethenyl]phenyl]amine (3). To a solution of tris(4-formylphenyl)amine ${ }^{62,63}$ (7) (98.8 $\mathrm{mg}, 0.3 \mathrm{mmol})$ and $4(400.5 \mathrm{mg}, 0.99 \mathrm{mmol})$ in anhyd $\mathrm{CH}_{2} \mathrm{Cl}_{2}(10 \mathrm{~mL})$ was added $t$-BuOK $(151.5 \mathrm{mg}, 1.35 \mathrm{mmol})$. The mixture was stirred at $20^{\circ} \mathrm{C}$ for $16 \mathrm{~h}$, then refluxed for 2 $\mathrm{h}$, and the solvent was removed under reduced pressure. After the addition of water, extraction with $\mathrm{CH}_{2} \mathrm{Cl}_{2}$, and drying $\left(\mathrm{Na}_{2}\right.$ $\mathrm{SO}_{4}$ ), the solvent was evaporated. The crude product was purified by column chromatography (heptane $/ \mathrm{CH}_{2} \mathrm{Cl}_{2}, 20: 80$ ) to yield $116 \mathrm{mg}(36 \%)$ of $3: \mathrm{mp} 88{ }^{\circ} \mathrm{C}$; ${ }^{1} \mathrm{H} \mathrm{NMR}(200.13 \mathrm{MHz}$, $\left.\mathrm{CDCl}_{3}\right) \delta 7.87$ and $7.65\left(\mathrm{AA}^{\prime} \mathrm{XX}^{\prime}, J_{\mathrm{AX}}=8.5,12 \mathrm{H}\right), 7.47$ and $7.14\left(\mathrm{AA}^{\prime} \mathrm{XX}^{\prime}, J_{\mathrm{AX}}=8.7,12 \mathrm{H}\right), 7.23(\mathrm{~d}, J=16.0,3 \mathrm{H}), 7.05$ $(\mathrm{d}, J=16.0,3 \mathrm{H}), 3.09(\mathrm{~m}, 6 \mathrm{H}), 1.73(\mathrm{~m}, 6 \mathrm{H}), 1.24(\mathrm{~m}, 30 \mathrm{H})$, $0.86(\mathrm{t}, J=6.5,9 \mathrm{H}) ;{ }^{13} \mathrm{C} \mathrm{NMR}\left(50.32 \mathrm{MHz}, \mathrm{CDCl}_{3}\right) \delta 147.1$, $142.8,137.1,132.1,131.5,128.5,128.0,126.7,125.4,124.3$, 56.3, 31.6, 28.9, 28.8, 28.2, 22.6, 22.5, 14.0; HRMS (LSIMS ${ }^{+}$, mNBA) calcd for $\mathrm{C}_{66} \mathrm{H}_{81} \mathrm{NO}_{6} \mathrm{~S}_{3}\left(\mathrm{M}^{+\bullet}\right) \mathrm{m} / \mathrm{z}, 1079.5226$, found 1079.5245. Anal. Calcd for $\mathrm{C}_{66} \mathrm{H}_{81} \mathrm{NO}_{6} \mathrm{~S}_{3}$ (1080.57): C, 73.36; H, 7.56; N, 1.30; S, 8.90. Found: C, 73.08; H, 7.49; N, 1.15; S, 9.08 .

2.2. Theoretical Approaches. We employed a blend of quantum-chemical approaches to model both linear and nonlinear spectra of the chromophores of interest. For the sake of simplicity, the alkyl solubilizing chains have been replaced by methyl groups. Solvation effects were neglected. Ground-state optimized geometries have been obtained using the Gaussian $98^{64}$ package. The geometry optimization was conducted using the $6-31 G^{*}$ basis set at the Hartree-Fock (HF) and hybrid density functional theory (DFT) B3LYP levels. The latter method represents one of the currently most accurate DFT functionals. For ground-state geometries, we previously found that the HF method is superior to the B3LYP approach by accurately reproducing the bond-length alternation parameter in similar conjugated systems when compared to experiment. ${ }^{65}$ Thus, all ground-state geometries used for discussion are obtained at the HF level.

Excited-state electronic structures (up to 20 excited states for each molecule) were calculated with Gaussian $98 .{ }^{64}$ It is wellknown that TD-HF lacks important electronic correlations, and therefore, excited states are systematically and significantly blueshifted with respect to experiments. In contrast, TD-DFT reproduces excited-state properties of many systems much better. ${ }^{66}$ However, pure and gradient-corrected DFT functionals do not reproduce charge-transfer states (HF does within a certain approximation). For example, standard GGA functionals tend to underestimate the excitation energies of charge-transfer states $^{67,68}$ because of spurious self-interaction. This can be partly cured by hybrid functionals such as $\mathrm{B}^{2} \mathrm{LYP}^{69}$ and was found to be very accurate for excited states in many molecular systems. ${ }^{66,70,71}$ Therefore, we used the TD-B3LYP/6-31G level of theory to investigate linear and nonlinear optical properties using the density matrix formalism for NLO responses as described in ref 72. This method has already demonstrated its accuracy for both linear and nonlinear spectra in different series of substituted chromophores. ${ }^{65,73,74}$

Vertical transition frequencies $\Omega_{g v}$, dipoles $\mu_{g v}$, and densities $\xi_{g v}$ are used ${ }^{72}$ to model both linear absorption and TPA spectra. The linear absorption at frequency $\omega$ is given by the imaginary part of

$$
\alpha(\omega)=\sum_{v} \frac{f_{g v}}{\Omega_{g v}^{2}-(\omega+i \Gamma)^{2}}
$$

where $\Gamma$ is the line width, and $f_{g v}$ is the oscillator strength associated with the $|g\rangle$ to $|v\rangle$ electronic transition. The TPA cross-section $\sigma_{2}(\omega)$ is related to the imaginary part of the thirdorder polarizability $\gamma(-\omega ; \omega, \omega,-\omega)$ by

$$
\sigma_{2}(\omega)=\frac{4 \pi^{2} \hbar \omega^{2}}{n^{2} c^{2}} L^{4} \operatorname{Im}\langle\gamma\rangle
$$

where $\hbar$ is Plank's constant, $c$ is the speed of light, $n$ is the refractive index of the medium, $L$ is the local-field factor, and

$$
\langle\gamma\rangle=\frac{1}{15}\left[3 \sum_{i} \gamma_{i i i i}+\sum_{j \neq i}\left(\gamma_{i i j j}+\gamma_{i j i j}+\gamma_{i j j i}\right)\right]
$$

is the orientational average of $\gamma$ following eq 6 in ref 75. $\gamma(-$ $\omega ; \omega, \omega,-\omega)$ has been calculated using the density matrix formalism as described in ref 72 and implemented in ref 65 . All comparisons with experiment for the amplitude of the responses are subject to an uncertainty in the choice of the linebroadening parameter, $\Gamma$. In all calculations, we used an empirical line width of $\Gamma=0.1 \mathrm{eV}$.

To model the fluorescence spectra, we used the TURBOMOLE $^{76}$ package to optimize molecular geometries for the lowest excited state at (TD-HF)/6-31G level. TD-B3LYP optimizations were not stable and often resulted in low-lying unphysical charge-transfer states with vanishing oscillator strengths (see previous discussion). ${ }^{67,68}$ Thus for all subsequent analysis, we will consistently use molecular geometries based on HF and TD-HF approaches and excited states calculated with 
the TD-B3LYP method. Namely, absorption and fluorescence points were treated at the TD-B3LYP/6-31G//HF/6-31G* and TD-B3LYP/6-31G//TD-HF/6-31G levels, respectively, in conventional quantum-chemical notation "single point//optimization level". At excited-state optimal geometry, transition frequencies $\Omega_{g e}^{(f)}$ and dipoles $\mu_{g e}^{(f)}$ correspond to the vertical fluorescence process and were used to calculate the radiative lifetime $\tau_{0}$ as ${ }^{77}$ (cgs units)

$$
\frac{1}{\tau_{0}}=\frac{4}{\hbar c^{3}\left(n^{2}+2\right)} \Omega_{g e}^{(f)^{3}} \mu_{g e}^{(f)^{2}}
$$

To analyze the nature of the excited states involved in the photophysical processes, we used natural transition orbital analysis of the excited states ${ }^{78,79}$ based on the calculated transition densities. This analysis offers the most compact representation of a given transition density in terms of an expansion into single-particle transitions. Wave functions for degenerate states in the octupolar specimen are defined by implementation of the Davidson diagonalization in Gaussian $98^{64}$ and differ from the "canonical" eigenfunctions $1 / \sqrt{6}\left(2 \phi_{1}\right.$ $\left.-\phi_{2}-\phi_{3}\right)$ and $1 / \sqrt{2}\left(\phi_{2}-\phi_{3}\right) \cdot{ }^{27}$ Figures showing molecular geometries and transition orbitals were obtained with XCrySDen. 80

To connect the photophysical properties of multibranched chromophores $\mathbf{2}$ and $\mathbf{3}$ to those of their single-branch counterpart 1, we use a Frenkel exciton model. ${ }^{81,82}$ This approach is applicable to molecular aggregates (such as molecular crystals, ${ }^{82}$ $H$ - and $J$-aggregates, ${ }^{83,84}$ and biological complexes ${ }^{85,86}$ ) and assumes an electrostatic interaction between monomers $(V)$, which is small compared to the typical transition energy ( $V \ll$ $\Omega$ ). The intermolecular coupling does not necessarily have to be of dipole-dipole nature, and the chromophores do not necessary have to be spatially separated (phenylacetylene dendrimers are examples of such cases ${ }^{87}$ ). As is the case for many conjugated molecules, the lowest excited state of monomer 1 with frequency $\Omega_{g e}$ (which is the origin of the excitonic manifold in multibranched chromophores) is expected to be responsible for the dominant contributions in the spectra. In particular, the Frenkel exciton Hamiltonian for chromophores $\mathbf{2}$ and $\mathbf{3}$ for the lowest excitonic manifold is given by

$$
H=\Omega_{g e} \sum_{i}^{2 \text { or } 3} B_{i}^{+} B_{i}+V \sum_{i, j \neq i}^{2 \text { or } 3} B_{j}^{+} B_{i}
$$

where $B_{i}^{+}\left(B_{i}\right)$ are the excitonic creation (annihilation) operators on branch $i$. After diagonalization of the Hamiltonian (5), ${ }^{27}$ the resulting splittings in the energies of the excitonic states are cartooned in Scheme 2, where $|\mathbf{g}\rangle$ denotes the ground state, and $|\mathbf{e}\rangle,\left|\mathbf{e}^{\prime}\right\rangle$, and $\left|\mathbf{e}^{\prime \prime}\right\rangle$ the excited states. The V-shaped character of $\mathbf{2}$ is responsible for the nonvanishing one-photon oscillator strength of the symmetric (higher-energy) state $2\left|\mathbf{e}^{\prime}\right\rangle$, even if $\mathbf{2}|\mathbf{e}\rangle$ is the most one-photon-allowed state. If $C_{3}$ symmetry is assumed for the three-branched system, a twofold degenerated first excited state $\left(|\mathbf{e}\rangle\right.$ and $\left.\left|\mathbf{e}^{\prime}\right\rangle\right)$ is obtained, which is both oneand two-photon-allowed, while excited state $\left|\mathbf{e}^{\prime \prime}\right\rangle$ is the only two-photon state allowed. These excited states, together with the ground state (the vacuum state with respect to the $B_{i}{ }^{+} B_{i}$ operators) can be inserted in the sum-over-states expression ${ }^{88}$ for $\gamma_{i j k l}$, and the TPA cross section is obtained through eqs 3 and 2.

2.3. Photophysical Methods. UV-vis spectra were recorded on a Jasco V-570 spectrophotometer. Steady-state and timeresolved fluorescence measurements were performed at room
SCHEME 2. Schematic Electronic-Level Diagram of the Single Branch (left) and of the Molecular Systems Built from Gathering Two (middle) or Three (right) Such Branches within the Excitonic Model ${ }^{a}$

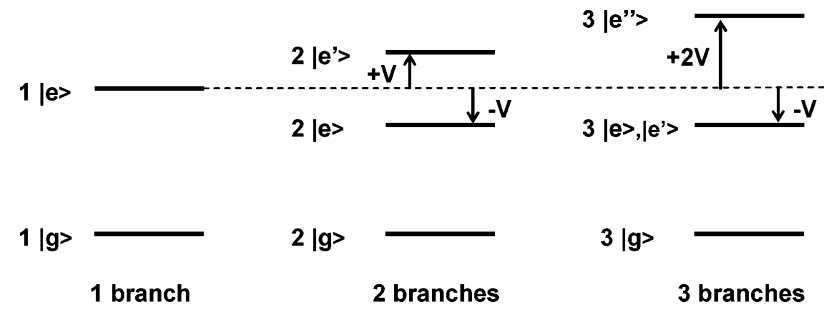

${ }^{a}|\mathbf{g}\rangle$ denotes the ground state, $|\mathbf{e}\rangle,\left|\mathbf{e}^{\prime}\right\rangle$, and $\left|\mathbf{e}^{\prime \prime}\right\rangle$ the excited states, and $V$ the coupling between adjacent branches. $C_{3}$ symmetry has been assumed for the three-branched system leading to a twofold degenerated first excited state $\left(|\mathbf{e}\rangle\right.$ and $\left.\left|\mathbf{e}^{\prime}\right\rangle\right)$. The structures of the excitonic eigenfunctions shown in Figure 7 can be clearly identified in the respective transition orbitals of an electron in all branched chromophores.

temperature in dilute solutions (ca. $10^{-6} \mathrm{M}$ ) using an Edinburgh Instruments (FLS 920) spectrometer in photon-counting mode. Emission spectra were obtained for each compound at $\lambda_{\mathrm{ex}}=$ $\lambda_{\max }(\mathrm{abs})$ with $A_{\lambda_{\mathrm{ex}}} \leq 0.1$ to minimize internal absorption. Fluorescence quantum yields were measured on degassed samples at room temperature; fluorescein in $0.1 \mathrm{~N} \mathrm{NaOH}$ was used as a standard (quantum yield $\Phi=0.90$ ). ${ }^{89}$ The lifetime values were obtained from the reconvolution fit analysis of the decay profiles with the F900 analysis software, and the fitting results were judged by the reduced $\chi^{2}$ value.

Two-photon excitation cross-sections of chromophores 1-3 were determined by investigating their TPEF in solution (concentration ca. $10^{-4} \mathrm{M}$ ). These measurements provide the TPEF action cross-section $\sigma_{2} \Phi$. The corresponding $\sigma_{2}$ values were derived by determining the fluorescence quantum yield $\Phi$ from standard fluorescence measurements. We emphasize that experiments were conducted in the femtosecond regime, thus preventing any contribution from linear nonresonant absorption or from excited-state absorption that is known to lead to artificially enhanced "effective" TPA cross-sections when measurements are conducted in the nanosecond regime.

TPEF measurements were conducted using a mode-locked Ti:sapphire laser operating between 700 and $1000 \mathrm{~nm}$ and delivering 80-fs pulses at $80 \mathrm{MHz}$, following the experimental protocol described in detail by $\mathrm{Xu}$ and Webb. ${ }^{90}$ The quadratic dependence of the fluorescence intensity on the excitation intensity was verified for each data point, indicating that the measurements were carried out in intensity regimes in which saturation or photodegradation do not occur. TPEF measurements were calibrated relative to the absolute TPEF action crosssection determined by $\mathrm{Xu}$ and Webb for fluorescein in water $(\mathrm{pH}=11)$ in the $690-1000-\mathrm{nm}$ range.$^{90,91}$ For each data point, an additional control was performed using the known TPEF action cross-section of rhodamine $\mathrm{B}$ in methanol. ${ }^{90,91}$ The experimental uncertainty amounts to $\pm 10 \%$.

\section{Linear Spectroscopy}

3.1. Experimental Results. The photophysical characteristics of chromophores 1-3 in toluene are summarized in Table 1. All the chromophores show an intense absorption band in the near-UV-blue-visible region (Figure $2 b$ ). The molar extinction coefficients are found to increase almost linearly with the number of branches, indicating a nearly additive behavior (Table 1). Gathering dipolar units (chromophore 1) via a common 
TABLE 1: Photophysical Data of Chromophores 1-3 (in toluene)

\begin{tabular}{|c|c|c|c|c|c|c|c|c|c|c|c|c|}
\hline & $\begin{array}{c}\lambda_{\mathrm{abs}}^{\text {exptl } a} \\
(\mathrm{~nm})\end{array}$ & $\begin{array}{c}\lambda_{\text {abs }}^{\text {calcd } b} \\
(\mathrm{~nm})\end{array}$ & $\begin{array}{c}\epsilon_{\max } \\
\left(\mathrm{cm}^{-1} \mathrm{~mol}^{-1} \mathrm{~L}\right)\end{array}$ & $\begin{array}{l}\text { fwhm } \\
\left(\mathrm{cm}^{-1}\right)\end{array}$ & $\begin{array}{l}\mu_{\mathrm{ge}} \\
(\mathrm{D})\end{array}$ & $\begin{array}{c}\lambda_{\mathrm{em}}^{\operatorname{exptl} d} \\
(\mathrm{~nm})\end{array}$ & $\begin{array}{c}\lambda_{\mathrm{em}}^{\mathrm{calcd}_{e}} \\
(\mathrm{~nm})\end{array}$ & $\begin{array}{l}\text { Stokes shift } \\
\left(\mathrm{cm}^{-1}\right)\end{array}$ & $\Phi^{f}$ & $\begin{array}{c}\tau^{g} \\
(\mathrm{~ns})\end{array}$ & $\begin{array}{c}\tau_{0}^{\text {exptl } h} \\
\text { (ns) }\end{array}$ & $\begin{array}{c}\tau_{0}^{\text {calcd }_{i}} \\
(\mathrm{~ns})\end{array}$ \\
\hline 1 & 392 & $400(423)$ & $2.9 \times 10^{4}$ & 3630 & 6.2 & 456 & 454 & 3580 & 0.58 & 1.4 & 2.5 & 1.5 \\
\hline 2 & 409 & $420(453)$ & $5.0 \times 10^{4}$ & 4250 & 8.9 & 459 & 480 & 2660 & 0.74 & 1.6 & 2.1 & 1.3 \\
\hline 3 & 410 & 419 (456) & $7.0 \times 10^{4}$ & 3595 & 10.4 & 463 & 486 & 2790 & 0.72 & 1.7 & 2.3 & 1.3 \\
\hline
\end{tabular}

${ }^{a}$ Experimental one-photon absorption maximum. ${ }^{b}$ Calculated one-photon vertical absorption maximum at TD-B3LYP/6-31G//HF/6-31G* (B3LYP/ 6-31G*) level. ${ }^{c}$ Half-bandwidth. ${ }^{d}$ Experimental one-photon emission maximum. ${ }^{e}$ Calculated one-photon vertical emission maximum at TD-B3LYP/ 6-31G //TD-HF/6-31G level. ${ }^{f}$ Fluorescence quantum yield determined relative to fluorescein in $0.1 \mathrm{~N} \mathrm{NaOH} .{ }^{g}$ Experimental fluorescence lifetime determined using time-correlated single-photon counting (TCSPC). ${ }^{h}$ Radiative lifetime derived from fluorescence quantum yield and lifetime values (experimental values). ${ }^{i}$ Radiative lifetime derived from quantum-chemical calculations using eq 4 (calculated at TD-B3LYP/6-31G //TD-HF/ 6-31G level).
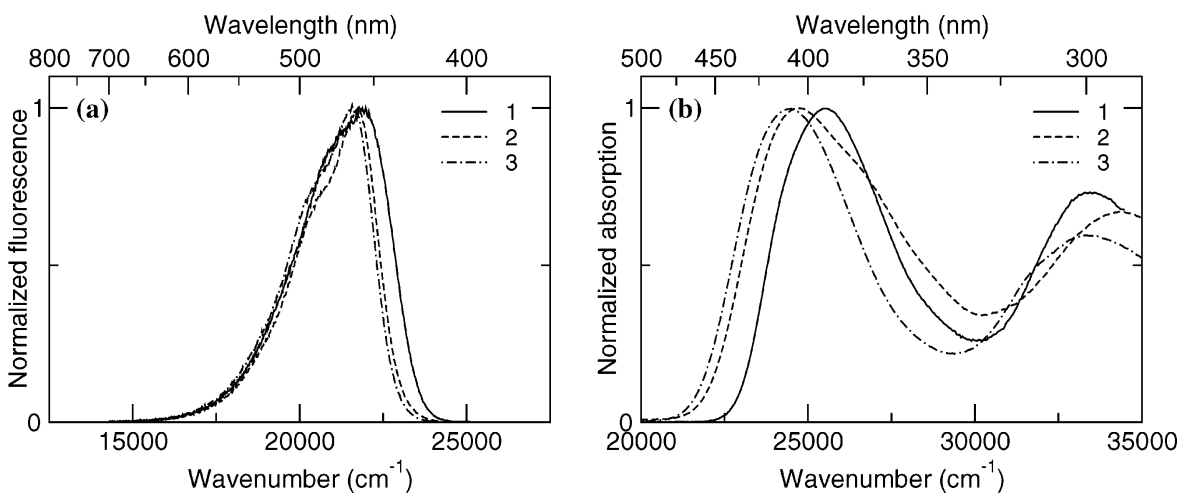

Figure 2. Normalized fluorescence (a) and absorption spectra (b) of chromophores $\mathbf{1}-\mathbf{3}$ in toluene.

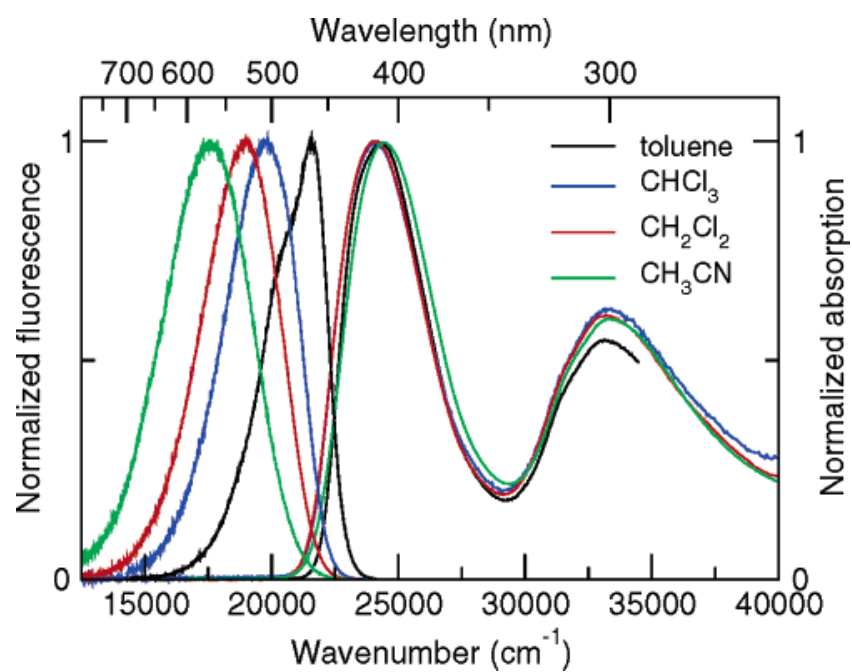

Figure 3. Solvatochromic behavior of chromophore 3.

electron-donating nitrogen to obtain either V-shaped (twobranched compound 2) or trigonal (three-branched compound 3) branched molecules leads to a slight red-shift of both the absorption and emission bands (Figure 2), with a more pronounced effect on absorption than on fluorescence spectra.

All three chromophores show a marked positive emission solvatochromism: increasing solvent polarity leads to a pronounced bathochromic shift of the emission band (Figure 3 and Supporting Information). Solvent polarity allows the tuning of the photoluminescence in a significant way, varying typically from blue in toluene (Table 1$)$ to green or yellow $\left(\lambda_{\text {em }}=541\right.$, 556 , and $570 \mathrm{~nm}$ for chromophores $\mathbf{1}, \mathbf{2}$, and $\mathbf{3}$, respectively) in acetonitrile. In contrast, no noticeable shift is observed for the absorption band (Figure 3 and Supporting Information). Accordingly, the Stokes shifts significantly increase with increasing solvent polarity. Such behavior is consistent with a stabilization of highly polar emitting excited states by polar solvents. In

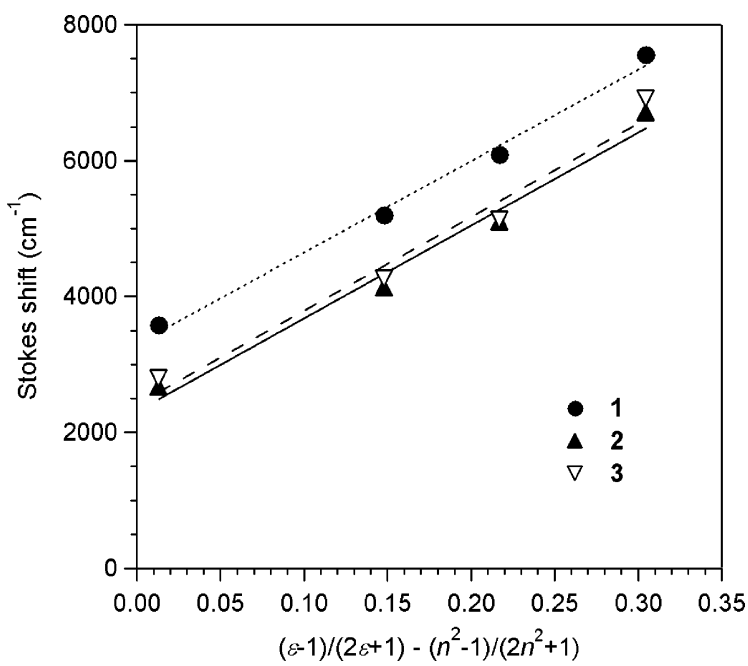

Figure 4. Lippert-Mataga correlations for chromophores 1-3.

addition, as shown in Figure 4, the solvatochromic behavior follows the Lippert-Mataga relationship ${ }^{92,93}$

$$
\tilde{v}_{\mathrm{abs}}-\tilde{v}_{\mathrm{em}}=2\left(\mu_{e e}^{(f)}-\mu_{g g}\right)^{2} \Delta f /\left(h c a^{3}\right)+\mathrm{const}
$$

where $\tilde{v}_{\text {abs }}$ and $\tilde{v}_{\text {abs }}$ are the wavenumbers of the absorption and fluorescence maxima, $h$ is the Planck constant, $c$ is the light velocity, $a$ is the radius of the solute spherical cavity, and $\Delta f$ is defined as

$$
\Delta f=(\epsilon-1) /(2 \epsilon+1)-\left(n^{2}-1\right) /\left(2 n^{2}+1\right)
$$

where $\epsilon$ is the dielectric constant and $n$ is the refractive index of the solvent.

The photoluminescence characteristics were found to depend on the dimensionality of the molecule. Even so, chromophores 1-3 have similar emission spectra (Figure 2a), but two- and three-branched chromophores $\mathbf{2}$ and $\mathbf{3}$ exhibit higher fluores- 

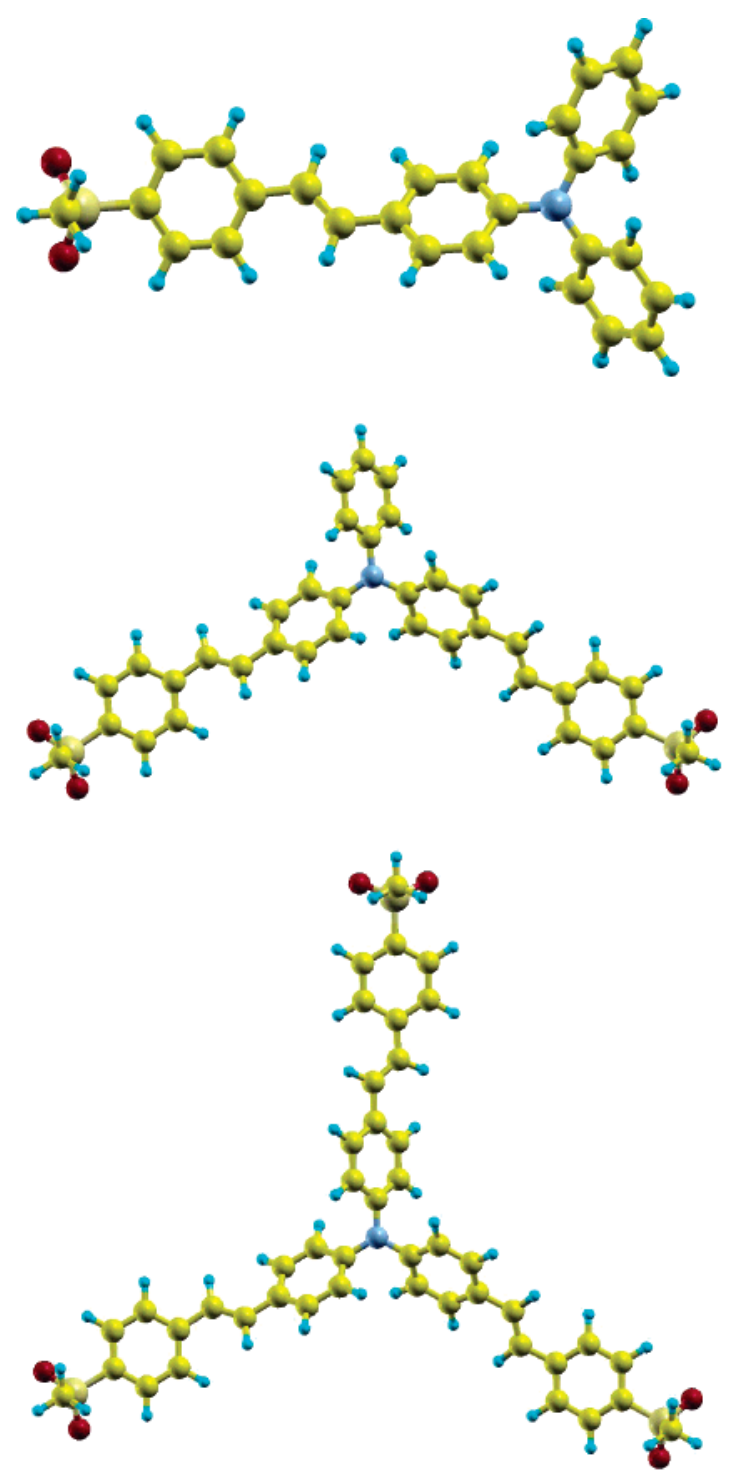

Figure 5. Optimized geometry of chromophores $\mathbf{1}-\mathbf{3}\left(\mathrm{HF} / 6-31 \mathrm{G}^{*}\right.$ level).

cence quantum yields than their one-dimensional dipolar analogue, 1 (Table 1). This seems to originate mainly from slower nonradiative decay. Interestingly, we also observe that the derived radiative lifetimes $\left(\tau_{0}=\tau / \Phi\right)$ are almost constant for the whole series (Table 1).

3.2. Discussion and Interpretation. Quantum Chemistry Results. Quantum-chemical calculations indicate that in chromophores $\mathbf{1}-\mathbf{3}$ the triphenylamine moiety adopts a propeller-shaped structure, the phenyl rings being twisted with respect to the trigonal planar nitrogen (dihedral angle smaller than $1^{\circ}$ ). The optimized ground-state geometries of $\mathbf{1 - 3}$ at the HF level are shown in Figure 5. HF results yield larger twist angles (between $38^{\circ}$ and $51^{\circ}$ ) than B3LYP results (between $35^{\circ}$ and $48^{\circ}$ ). These results are consistent with crystallographic data..$^{94}$ The conjugated stilbenyl branches are found to be almost planar at the B3LYP level, while the two phenyl rings are substantially twisted at the HF level with phenyl-vinyl twist angles between $18^{\circ}$ and $23^{\circ}$. The bond-length alternation parameter defined as the difference between single and double bonds on the vinyl bridge is $0.15 \AA$. This parameter reflects the degree of conjugation along the molecular backbone. ${ }^{95}$ Overall, all branches in $\mathbf{2}$ and $\mathbf{3}$ have ground-state geometries similar to the geometry of the parent molecule, 1 . This implies that branched compounds have higher symmetries (e.g., $C_{3}$ for chromophore 3).

However, the excited-state geometries are different. First of all, the structure of the stilbenyl branch in $\mathbf{1}$ becomes planar with a vanishing bond-length alternation parameter. This is a generic phenomenon observed in many extended molecular systems such as conjugated polymers. ${ }^{95,96}$ The structures of multibranched chromophores $\mathbf{2}$ and $\mathbf{3}$ do not retain their symmetries: roughly, one branch adopts a geometry similar to the excited-state geometry of molecule $\mathbf{1}$, whereas the other branch(es) remain(s) in the ground-state geometry. This is an indication that the fluorescence of $\mathbf{2}$ and $\mathbf{3}$ originates from an excitation localized on a single branch rather than from a fully delocalized state. It should be stressed that we do not observe any level crossing in these molecules. The nature of the lowest excited state does not change upon vibrational relaxation as evidenced by the examination of the appropriate transition orbitals (see following text). Moreover, the other excited states are always well-separated from the state in question during geometry optimizations. Thus, this scenario is completely different from the formation of the so-called twisted intramolecular charge-transfer states, which may possibly lead to the observation of dual fluorescence. The latter is linked to the presence of a conformational degree of freedom having multiple minima for the ground and excited states, as previously reported in several molecular systems (e.g., in $\mathrm{DMABN}^{97,98}$ ).

Some calculated electronic quantities are given in Table 2. The global agreement between experiment and theory is better emphasized in Figure 6, where the experimental absorptions of chromophores $\mathbf{1}$ and $\mathbf{2}$ are compared to the spectra calculated for the HF and B3LYP geometries. As expected, ${ }^{65}$ the best agreement is obtained for HF geometries, which will be used for discussion.

Frenkel Exciton Model. The red-shift of the absorption band at increasing branching character (Figure 2b) is indicative of electrostatic coupling between the dipolar branches that can be described using the Frenkel exciton model (Scheme 2). The first excited state basically corresponds to an electron transfer from the triphenylamine moiety to the conjugated branch(es) and is common to all three molecules (Figure 7). The two-branched, $\mathrm{V}$-shaped chromophore $\mathbf{2}$ is found to display the largest halfbandwidth. This can be qualitatively explained by the exciton model, which predicts the presence of two splitted excited states (separated by an energy of $2 V$; see Scheme 2), both being onephoton-allowed because of the angle formed between the two interacting branches. The value of the angle in particular allows us to predict that the state at the bottom of the exciton band is more allowed, carrying the greatest oscillator strength $(3 / 4$ of the total for an angle of $120^{\circ}$ ). This is nicely confirmed by the following procedure: subtracting from the normalized absorption spectra of the V-shaped compound the absorption band of the dipolar compound (after normalization and an energy shift as to have coincident maximum positions) leads to a residual band with maximum at about $372 \mathrm{~nm}$ (see Supporting Information), that is, almost symmetrically displaced from the monomer band with respect to the other (lowest-energy) contribution. This means that the splitting is symmetric with respect to the first excited state of the monomer, as predicted by the Frenkel exciton model (Scheme 2). The deduced band also allows us to derive a crude estimate of the ratio between the oscillator strength corresponding to the first and second excited states $\left(f_{\mathrm{e}} / f_{\mathrm{e}^{\prime}}\right)$ of about 4. This estimate is in very good agreement with that derived from TD-DFT calculations based on the HF geometries, as they lead to $f_{\mathrm{e}}=1.12$ and $f_{\mathrm{e}^{\prime}}=0.26$. Thus, calculations 
TABLE 2: Theoretical Results for Chromophores 1-3 Obtained at TD-B3LYP/6-31G//HF/6-31G* and TD-B3LYP/6-31G// TD-HF/6-31G Levels ${ }^{a}$

\begin{tabular}{ccccccccc}
\hline state & $1 \mathrm{e}$ & $1 \mathrm{e}^{(\mathrm{f})}$ & $2 \mathrm{e}$ & $2 \mathrm{e}^{(\mathrm{f})}$ & $2 \mathrm{e}^{\prime}$ & $3 \mathrm{e}^{\prime} / 3 \mathrm{e}^{\prime}$ & $3 \mathrm{e}^{(\mathrm{f})}$ & $3 \mathrm{e}^{\prime \prime}$ \\
\hline$\Omega_{g e}{ }^{b}(\mathrm{eV})$ & 3.10 & 2.73 & 2.95 & 2.58 & 3.23 & 2.96 & 2.55 & 3.36 \\
$\mu_{g e}{ }^{c}(\mathrm{D})$ & $8.2(x)$ & $11.7(x)$ & $10.0(x)$ & $12.9(x)$ & $4.6(y)$ & $9.3(x y)$ & $12.8(x)$ & 0 \\
$\mu_{g g}{ }^{d}(\mathrm{D})$ & $7.3(x)$ & $10.4(x)$ & $6.7(y)$ & $8.0(y)$ & $6.7(y)$ & 0 & $1.9(x)$ & 0 \\
$\mu_{e e}{ }^{e}(\mathrm{D})$ & $25.8(x)$ & $19.2(x)$ & $14.0(y)$ & $13.1(y)$ & $13.8(y)$ & $11.9(x y)$ & $8.9(x)$ & 0
\end{tabular}

${ }^{a}$ Superscript (f) denotes the lowest excited-state optimal geometry corresponding to fluorescence. Dipole moments are given only as contributions on the $x y$ plane, because contributions along the $z$ axis are only relevant to local dipole moments due to $\mathrm{SO}_{2} \mathrm{Me}$ terminal groups (3-4 $\mathrm{D}$ per group). Beyond the modulus of each dipole moment, the main polarization direction is indicated in parentheses. ${ }^{b}$ Transition frequency. ${ }^{c}$ Transition dipole moment. ${ }^{d}$ Ground-state dipole moment. ${ }^{e}$ Excited-state dipole moment.
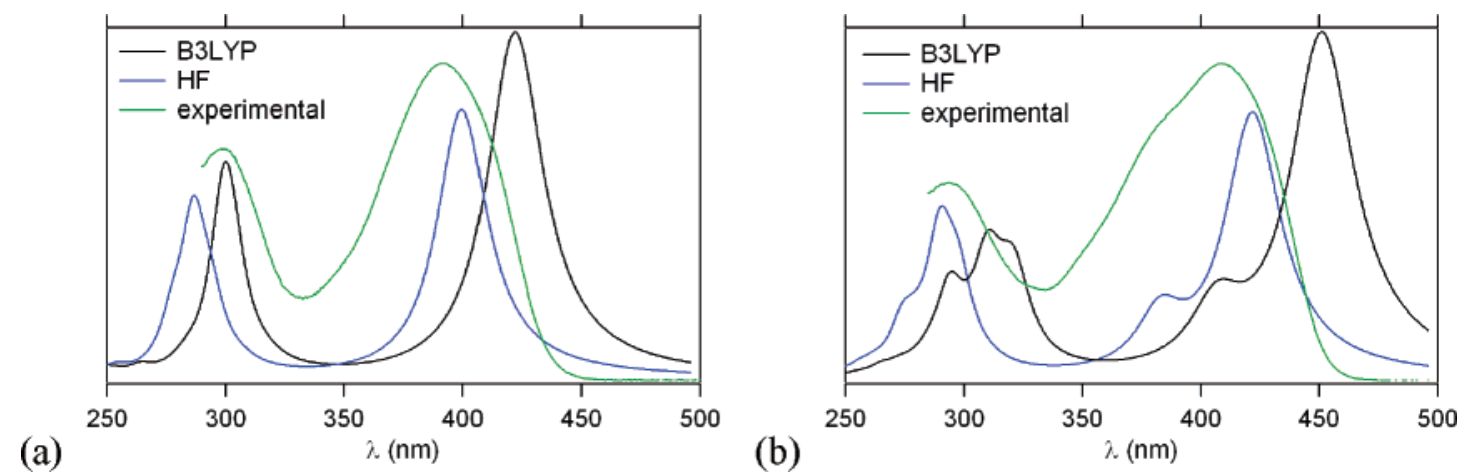

Figure 6. Calculated and experimental absorption of chromophores 1 (a) and 2 (b).

confirm that the broad and asymmetric absorption band of compound 2 (Figures 2 and 6) results from the overlap of two close electronic transitions, in correlation with the presence of two low-lying excited states. This can also be appreciated by comparing the absorption and fluorescence spectra of chromophore 2, which are far from being mirror images, as is instead almost the case for chromophores $\mathbf{1}$ and $\mathbf{3}$. From both experimental and theoretical results, the coupling constant $V$ is estimated to be $\sim 0.14 \mathrm{eV}$. The $V$ values obtained from calculations vary between 0.133 and $0.147 \mathrm{eV}$ (see Table 2). This same value can be obtained as a result of point-dipole interactions by imposing a distance of $8 \AA$ between the (pointlike) transition dipole moments. Considering the geometry of the branched structures, this distance corresponds to a length of $\sim 9 \AA$ for each dipolar branch, which is a reasonable estimate, in accord with what can be deduced from the optimized geometries and from the analysis of solvatochromic data (see following text).

In the three-branched chromophore, $\mathbf{3}$, for which the first two exited states are degenerate $\left(f_{\mathrm{e}}=f_{\mathrm{e}^{\prime}}=0.97\right)$, the third excited state is predicted to have vanishing oscillator strength $\left(f_{\mathrm{e}^{\prime \prime}}=\right.$ $0.0)$. As a matter of fact, the first absorption band of chromophore $\mathbf{3}$ does not show any spectral broadening due to the presence of additional excitonic states. This observation also confirms that chromophore $\mathbf{3}$ possesses a threefold symmetry axis. The energy difference between the degenerate first two excited states and the first excited state of the monomer leads again to a coupling constant $V$ of about $0.14 \mathrm{eV}$. This value is again in full agreement with those deduced from TD-DFT calculations (see Table 2). With the triphenylamine moiety imposing similar angles (ca. $120^{\circ}$ ) and distances between branches in chromophores $\mathbf{2}$ and $\mathbf{3}$, it is not surprising to find similar estimates for the coupling constant $V$. The position of the third excited state of chromophore $\mathbf{3}$, which is one-photon forbidden but two-photon-allowed, is thus predicted to show up at $0.42 \mathrm{eV}$ (Scheme 2) above the first two excited states (i.e., at $720 \mathrm{~nm}$ for two-photon excitation).

Dipole Moments and Charge Transfer. All chromophores investigated in the present work show a common feature: they exhibit large Stokes shifts. This indicates that significant reorganization takes place after excitation prior to emission. Such behavior can be related to the electronic redistribution occurring upon excitation. The ground and lowest-energy excited electronic states of push-pull molecules are often described as a combination of neutral and zwitterionic basis states represented by the corresponding molecular resonance forms. ${ }^{99,100}$ Calculations reveal that pronounced intramolecular charge transfer occurs within the chromophores upon excitation. Indeed, the transition orbitals of the first excited state(s) shown in Figure 7 reveal pronounced electronic density shifts from the triphenylamine moiety to the conjugated branch(es) in all three chromophores. Thus, the ground state is predominantly composed of the neutral form, while the excited state has a greater zwitterionic character (as also confirmed by the calculated bondlength alternation). However, all these states are not pure chargetransfer ones (with electron and hole well-separated spatially), but rather, states which undergo electron density redistribution from the donor to the acceptor and thus have only partial chargetransfer character. This further justifies usage of the hybrid DFT approaches. We further note that each excited state in question can be well-represented as a transition between a single dominant pair of transition orbitals (with $95 \%$ or more contribution).

The directional intramolecular charge transfer leads to a significant dipolar character of the first excited state for chromophores $\mathbf{1}$ and $\mathbf{2}$. In particular, calculations on molecule 1 predict a 25.8-D dipole moment in the excited state, compared to 7.3-D ground-state dipole. Excited-state relaxation reduces (increases) the dipolar character in the excited (ground) state (Table 2), and subsequently, the ratio of mixing between the neutral and zwitterionic states changes. This leads to an increase of the overlap between the electron and hole wave functions and a larger emitting transition dipole moment (11.7 D) compared to absorption (8.2 D). Whereas chromophore $\mathbf{1}$ is mainly dipolar, chromophore $\mathbf{2}$ combines dipolar and quadrupolar characteristics. This quadrupolar character shows up in the second excited state. For symmetry reasons, chromophore 3 undergoes upon excitation a two-dimensional intramolecular 


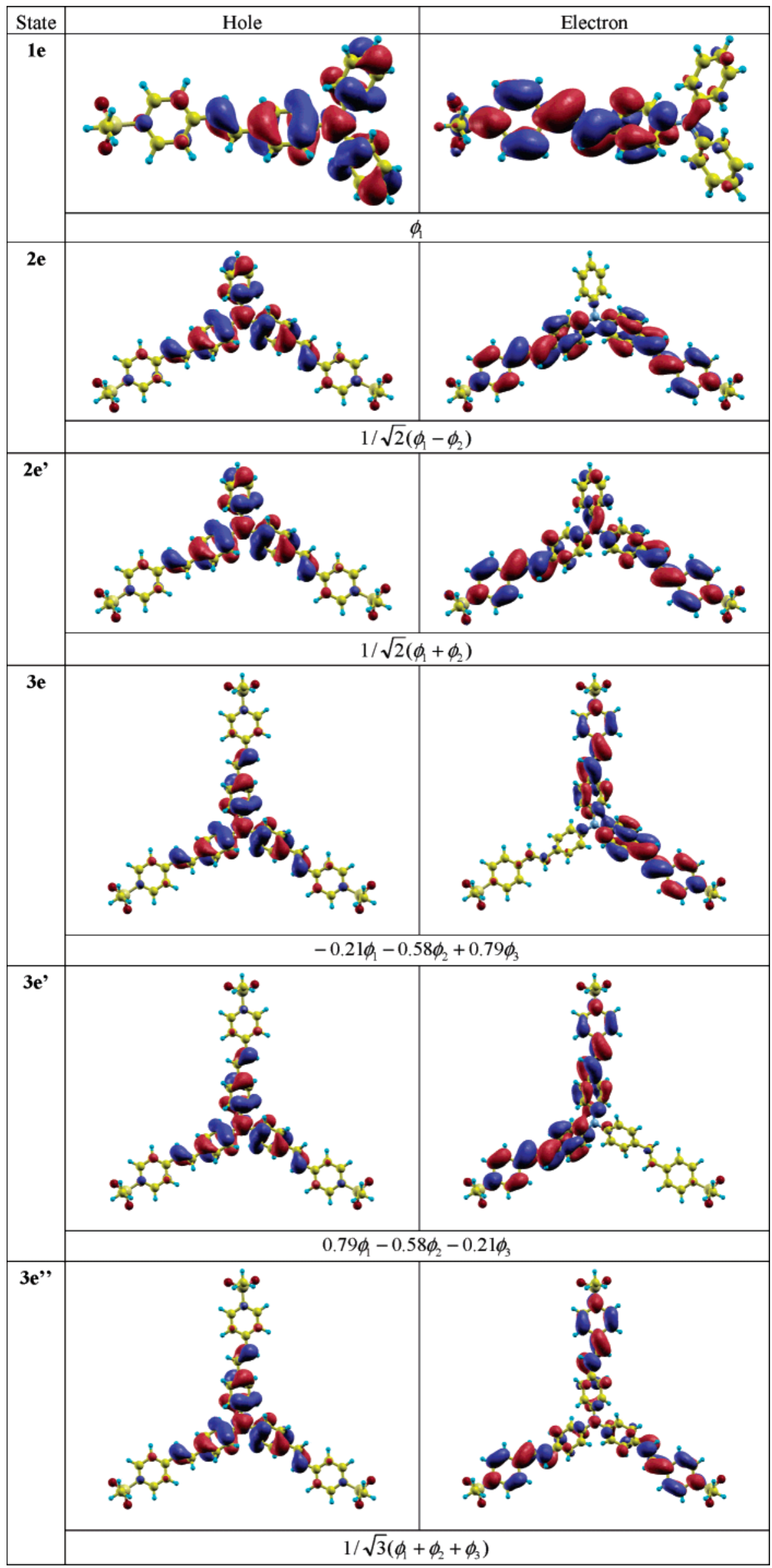

Figure 7. Natural transition orbitals of chromophores $\mathbf{1 - 3}$ (absorption). State labeling is given in Scheme 2. 


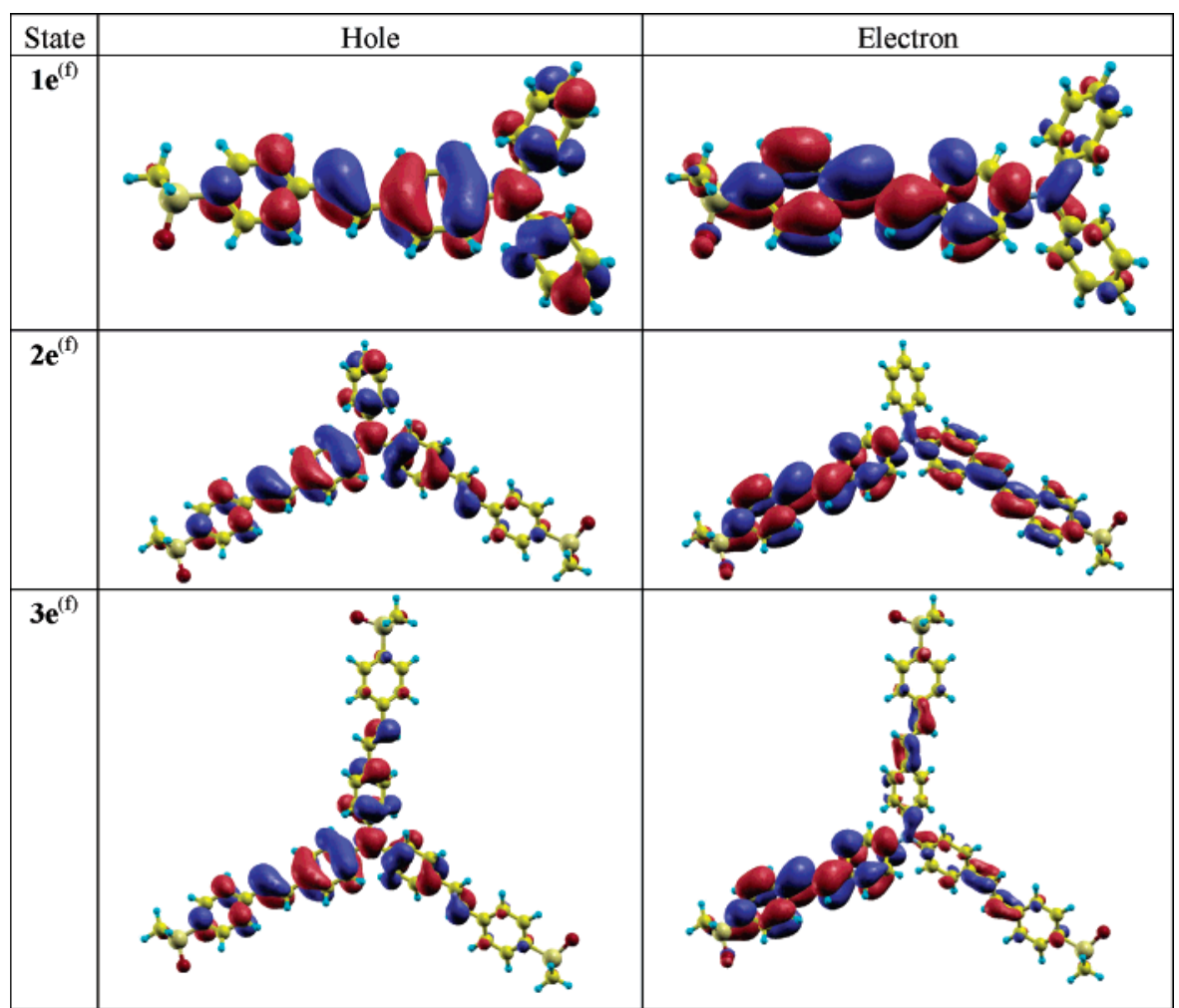

Figure 8. Natural transition orbitals of chromophores $\mathbf{1 - 3}$ (emission).

charge transfer from the trigonal core toward the branches (Figure 7). Even though the overall molecular dipole moments of the ground and excited states are considerably smaller in the branched structures compared to the monomer due to symmetry (Table 2), their local dipole moments on the emitting branch are very similar, which leads to large Stokes shifts.

As demonstrated in Figure 4, the Lippert-Mataga relation for the dependence of the Stokes shift on solvent polarity holds true for all the chromophores, and even more, the slope of the Stokes shift versus the polarity descriptor $\Delta f$ is the same for all of them. The good linearity of the trends in Figure 4 suggests that the effective mesomeric dipole moment $\left(\mu_{e e}^{(f)}-\mu_{g g}\right)^{\text {eff }}$ is almost constant at varying solvent polarity, in the studied polarity range. The fact that the slope is the same for all the compounds of the series is again an indication that the emitting excited state has the same nature in all cases (i.e., that fluorescence stems from an excited state localized on one branch). Otherwise, a sensible decrease of the slope would be observed along the series $\mathbf{1} \rightarrow \mathbf{2} \rightarrow \mathbf{3}$ because of the increased dimension of the solvent cavity. Thus, even though formally calculated total permanent dipole moments in the branched structures are reduced compared to the parent dipole monomer because of symmetry cancellation effects, the underlying wave function of the emitting excited state interacts primarily with the dipole moment of the single branch where it is localized. Thus, the effective difference $\left(\mu_{e e}^{(f)}-\mu_{g g}\right)^{\text {eff }}$ is essentially the same for all molecules, whereas the total magnitude of $\left(\mu_{e e}^{(f)}-\right.$ $\mu_{g g}$ ) substantially decreases with increasing branching. If the cavity radius is fixed at one-half of the estimated distance between the donor and acceptor moieties on the branch (i.e., $a$ $\approx 5 \AA$ ), a value of $\sim 13 \mathrm{D}$ is obtained for $\mu_{e e}^{(f)}-\mu_{g g}$, which is in good agreement with the values obtained by theoretical calculations (Table 2). Even by using a more refined ellipsoidal cavity model (which might be more appropriate for such elongated molecules) including shape and dipole position correction factors ${ }^{101}$ and taking into account the total length of the dipolar molecule (cavity radius $\approx 7 \AA$ ), we derive a change of the dipole moment of about $14 \mathrm{D}$.

Excited-State Localization. The experimental radiative lifetimes (obtained as $\tau_{0}=\tau / \Phi$ ) are almost constant for the whole series (Table 1), also confirming that the emission does not stem from a delocalized excited state in branched molecules. Otherwise, a decrease of $\tau_{0}$ would be expected as a result of the linear increase in the molar extinction coefficients with the number of branches. ${ }^{102}$ The theoretical computations of radiative lifetime (Table 1) reproduce trends observed in experiment but, however, underestimate the experimental values. ${ }^{103}$ While the small decrease in measured excited-state radiative lifetime with increasing branched character $(\mathbf{1} \rightarrow \mathbf{2} \rightarrow \mathbf{3}$ ) is well within the experimental incertitude, the slight decrease given by the calculations is probably due to slightly larger "emitting" transition dipoles in the branched structures (12.8 D) compared to $11.7 \mathrm{D}$ in 1 (Table 2). This indicates a minor delocalization of the emitting excited states in $\mathbf{2}$ and $\mathbf{3}$ (Figure 8), which may be further reduced by the solvation effect and/or the temperature bath, left unaccounted in our calculations.

As a matter of fact, transition orbitals representing the emitting state (Figure 8) clearly show a localization of the excitation on one branch, compared to the respective absorbing states (Figure 7). The very similar nature of the emitting excited states for all the chromophores is also confirmed by the very similar fluorescence frequencies and by the trend of the Stokes shift: within the Frenkel exciton model, if one assumes that the emitting excited state has the same nature and energy for all three chromophores, the magnitude of the Stokes shift is predicted to be reduced by $V(0.14 \mathrm{eV})$ from chromophore 1 to chromophores $\mathbf{2}$ and $\mathbf{3}$. This is close to being the case (Table 1). The reported calculations demonstrate that localization of the excitation is mainly due to molecular vibrations. This trend has a sounding counterpart in the Frenkel exciton model, where it is well-known that vibrational relaxation introduces diagonal disorder, which leads to localization of the excitation itself. ${ }^{104}$ 


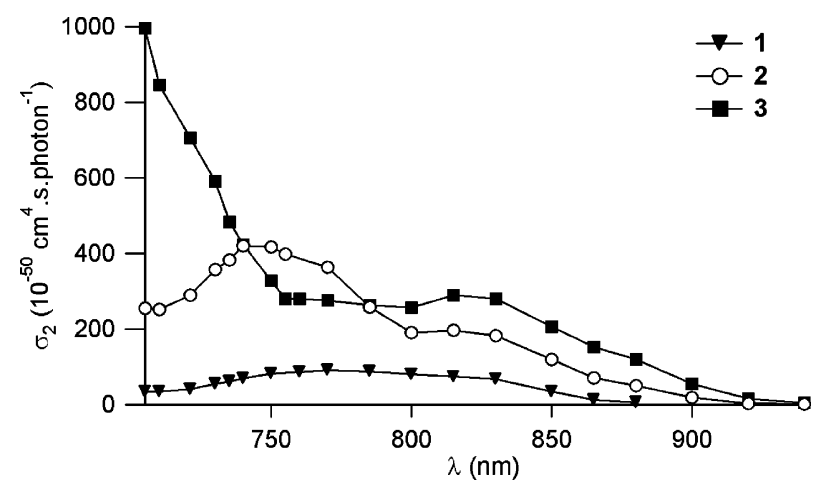

Figure 9. Two-photon excitation spectra $\left(\sigma_{2}\right)$ of molecules $\mathbf{1}-\mathbf{3}$ in toluene.

Solvation interaction can play an important role too, but in our case, the non-dipolar nature of the chosen solvent (toluene) rules out a major effect due to the solvent. Practically, calculations for the excited state predict an almost complete localization of the excitation as induced by nuclear relaxation only.

Thus, both theory and experiment consistently predict localization of the emitting state on a single branch. This is a general feature of the class of systems studied here. Actually, this phenomenon has also been reported for other octupolar trigonal derivatives, as proven by analogous photophysical studies 105,106 and time-resolved fluorescence anisotropy measurements. ${ }^{107,108}$ In particular, the latter technique estimates the characteristic time scale for nuclear relaxation to be on the order of few picoseconds. Excitation localization to a single monomer upon vibrational relaxation has also been predicted by recent theoretical investigations ${ }^{79,96}$ in other coupled aggregates composed by conjugated molecules. All these observations suggest that the phenomenon of localization of excitation is a quite common feature in interacting multichromophoric systems, where photoexcited individual branches undergo a significant vibrational relaxation and, in particular, in systems where the excited states are characterized by (partial) charge-transfer character. Bardeen and co-workers recently reported results on photoexcitation dynamics in small phenylacetylene dendrimers, ${ }^{109,110}$ where quantum confinement ensures strong participation of the branching core and a strong interaction among the branches in all excited states. In these systems, in addition to the dipolar interaction (Förster limit), there exists a considerable throughspace exchange interaction (Dexter mechanism). In the absorbing state, the interaction among branches cancels out for symmetry reasons, resulting in a very small total excitonic coupling. In contrast, vibrational relaxation (which leads to a localized state in our study) breaks the symmetry and the abovedescribed cancellation, which results in a large excitonic coupling for the emitting state in the case of small molecules. These observations are complementary to our findings.

\section{Two-Photon Absorption}

4.1. Experimental Results. The TPA spectra of chromophores $\mathbf{1 - 3}$ are shown in Figure 9. The first TPA maximum of the two- and three-branched chromophores $\mathbf{2}$ and $\mathbf{3}$ is red-shifted with respect to that of the dipolar chromophore, $\mathbf{1}$, as a result of the aforementioned exciton splitting (Scheme 2). A comparison between one-photon absorption (OPA) and (rescaled) TPA spectra is given in Figure 10, to emphasize the correspondence between the one- and two-photon-allowed transitions. In particular, for the dipolar chromophore, 1, (panel a), the only band in the visible region is both one- and two-photonallowed. For chromophore $\mathbf{2}$ (panel b), the slightly allowed

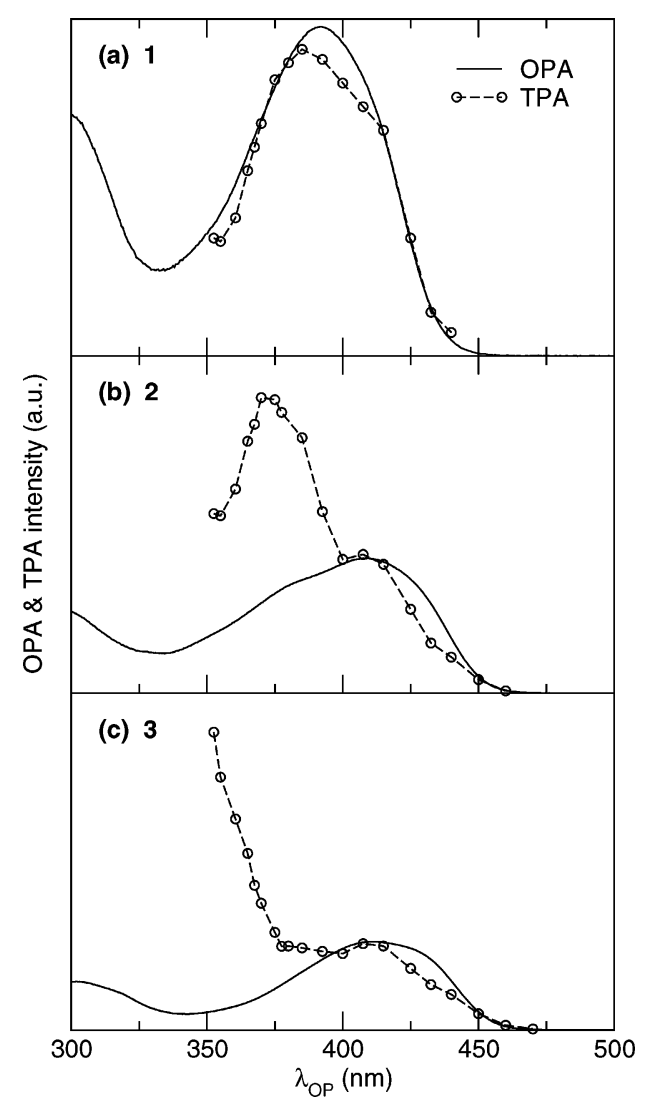

Figure 10. Experimental one-photon absorption spectra (continuous lines) and rescaled two-photon excitation spectra (symbols + dashed lines) of chromophores $\mathbf{1 - 3}$ in toluene.

higher-energy one-photon visible band (due to excitonic coupling) becomes the dominant contribution in the two-photon spectrum. For compound $\mathbf{3}$ (panel c), again the one-photon visible band is also two-photon-allowed, but the dominant contribution is due to the higher-energy excited state, which appears because of the electronic coupling between the branches.

The measured TPA cross-section peak values increase with increasing number of branches, but for consistently comparing the responses of the components of the series, some normalization criterion must be set. A first normalization procedure is based on the molecular weight, as to obtain a relevant figure of merit for applications such as optical limitations. The examination of TPA values normalized following this criterion (i.e., $\sigma_{2} /$ MW where MW is the molecular weight) at the first local maxima gives preliminary clues to the branching effect: we observe that branched chromophores $\mathbf{2}$ and $\mathbf{3}$ show larger normalized TPA values than the one-dimensional chromophore, 1 (Table 3). This slight cooperative enhancement is similar for the two- and three-branched derivatives (about 1.51.6). Furthermore, branched chromophores 2 and $\mathbf{3}$ show larger normalized TPA values $\left(\sigma_{2} / \mathrm{MW}\right)$ than dipolar chromophore 1 in the whole spectral range, indicating that the branching approach is a valid strategy for obtaining materials with increased TPA figures of merit.

Another normalization criterion can be chosen by simply rescaling the TPA response for the number of branches. This analysis yields better information on the intrinsic charge symmetry and branching effect, allowing one to distinguish additive behavior from cooperative effects. As shown in Figure 11, the so-normalized TPA responses of branched compounds always show an enhancement with respect to the dipolar analogue. This enhancement is also wavelength-dependent. In particular, it is weak near the first TPA maxima (i.e., close to 
TABLE 3: Two-Photon Absorption Data of Chromophores 1-3 (in toluene)

\begin{tabular}{|c|c|c|c|c|c|c|c|c|c|c|}
\hline \multirow[b]{2}{*}{ cpd } & \multirow[b]{2}{*}{$N_{\mathrm{eff}^{a}}^{a}$} & \multirow[b]{2}{*}{$2 \lambda_{\text {abs }}(\mathrm{nm})$} & \multirow[b]{2}{*}{$\lambda_{\mathrm{TPA}}^{\max 1}(\mathrm{~nm})$} & \multirow[b]{2}{*}{$\lambda_{\text {TPA }}^{\max 2}(\mathrm{~nm})$} & \multicolumn{2}{|c|}{$\sigma_{2}^{b}(\mathrm{GM})$} & \multicolumn{2}{|c|}{$\sigma_{2} / N_{\text {eff }}(\mathrm{GM})$} & \multicolumn{2}{|c|}{$\sigma_{2} / \mathrm{MW}\left(\mathrm{GM} \mathrm{g}^{-1} \mathrm{~mol}\right)$} \\
\hline & & & & & at $\lambda_{\text {TPA }}^{\max 1}$ & at $\lambda_{\text {TPA }}^{\max 2}$ & at $\lambda_{\text {TPA }}^{\max 1}$ & at $\lambda_{\text {TPA }}^{\max 2}$ & at $\lambda_{\text {TPA }}^{\max 1}$ & at $\lambda_{\mathrm{TPA}}^{\max 2}$ \\
\hline 1 & 18.1 & 784 & 770 & & 90 & & 4.97 & & 0.17 & \\
\hline 2 & 23.4 & 818 & 815 & 740 & 195 & 420 & 8.33 & 17.95 & 0.24 & 0.52 \\
\hline 3 & 27.7 & 820 & 815 & $\leq 705$ & 290 & $>995$ & 10.46 & 35.92 & 0.27 & $>0.92$ \\
\hline
\end{tabular}

${ }^{a}$ Effective number of $\pi$ electrons in the conjugated systems. ${ }^{111}{ }^{b}$ TPA cross-sections; $1 \mathrm{GM}=10^{-50} \mathrm{~cm}^{4} \mathrm{~s}$ photon ${ }^{-1}$; TPEF measurements were performed using a mode-locked Ti:sapphire laser delivering 80 -fs pulses at $80 \mathrm{MHz}$, calibrated with fluorescein.

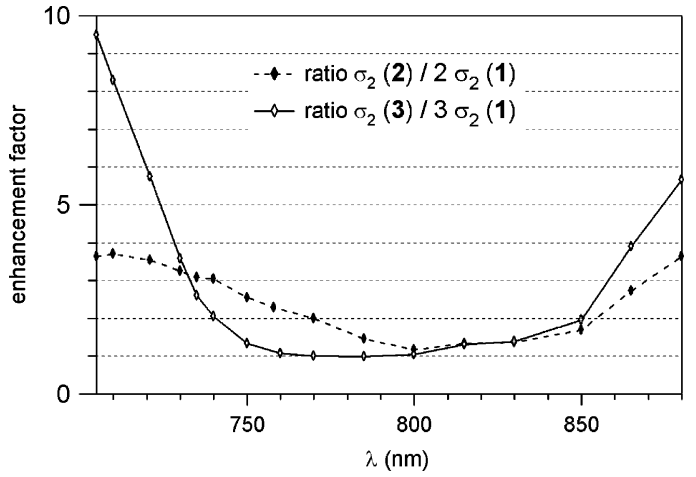

Figure 11. Wavelength dependence of branching effect on TPA.

$800 \mathrm{~nm}$ ) but significantly increases in the low-energy edge of the TPA band for both chromophores $\mathbf{2}$ and $\mathbf{3}$. This enhancement is mainly related to the red-shift of the lowest-energy one-photon absorption band that results from the coupling between dipolar branches.

It is interesting to note that related compounds were recently shown to lead to only a slight enhancement of their effective TPA cross-sections (derived from nonlinear transmission measurements conducted in the nanosecond regime) close to 800 $n m .{ }^{31}$ The TPEF experiments reported here provide experimental evidence that the TPA enhancement is strongly wavelengthdependent. The TPA enhancement also significantly increases close to the visible region (Figure 11). Chromophore 2, which combines dipolar and quadrupolar contributions, is the most efficient of the molecules in terms of normalized TPA in the 740-800 $\mathrm{nm}$ region, where a second and more intense TPA band appears (Figure 9). This band is directly related to the contribution of the higher excited-state lying close to the first excited state as a result of the coupling between the dipolar branches (Scheme 2). The energy gap between the first ( 815 $\mathrm{nm})$ and second $(740 \mathrm{~nm})$ TPA maxima amounts to $0.30 \mathrm{eV}$. This splitting value is again consistent with the coupling of 0.14 $\mathrm{eV}$ derived from the one-photon absorption bands using the Frenkel exciton model. Moreover, we observe that the maximum TPA cross-section of the V-shaped chromophore, 2 , is found to be nearly 5 times larger than that of the dipolar model, $\mathbf{1}$ (Table 2), revealing a cooperative enhancement (i.e., $\sigma_{2 \max }(n) /$ $\left.n \sigma_{2 \max }(\mathbf{1})\right)$ of about $200 \%$, which is definitely larger than experimental error.

The octupolar chromophore, $\mathbf{3}$, leads to the highest TPA normalized efficiency close to the visible red region (i.e., at $700 \mathrm{~nm}$ ). In this spectral region, the TPA cross-section is found to be more than 10 times larger than that of the peak TPA of the dipolar branch (Table 3), revealing a cooperative enhancement of more than $300 \%$, again much larger than experimental error. This indeed shows that assembling the dipolar chomophores of type $\mathbf{1}$ via the donating moiety in a trigonal structure is a very efficient route toward enhanced TPA.

4.2. Discussion and Interpretation. In Figure 12, the experimental TPA spectra (circles) are compared with calculated results. Dotted lines correspond to spectra obtained in the

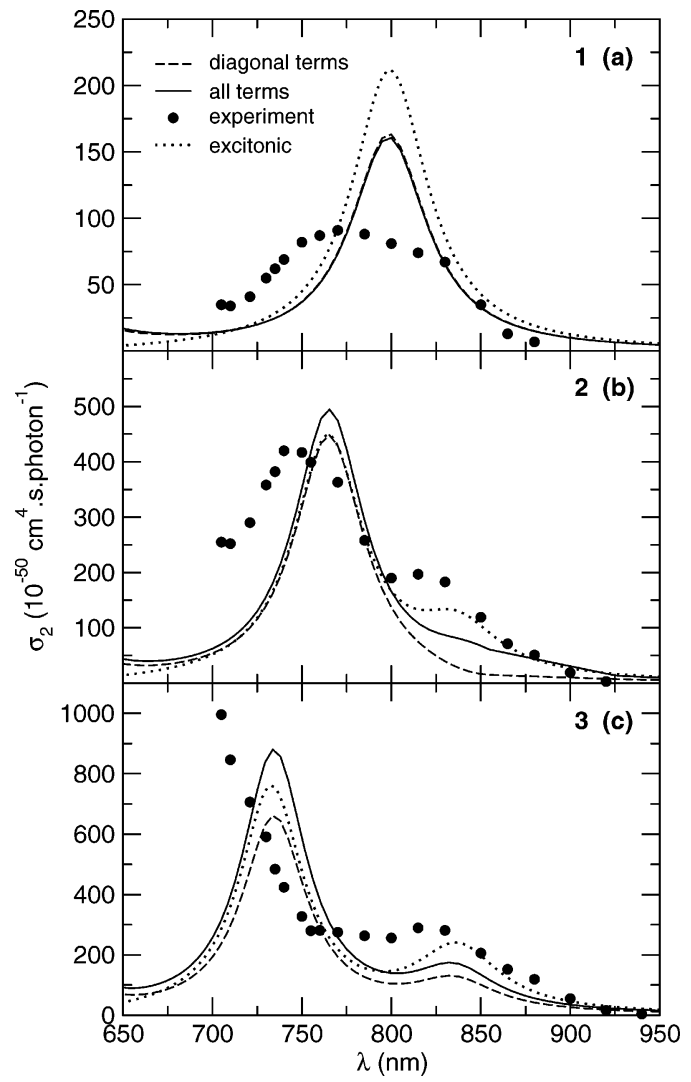

Figure 12. Calculated (lines) and experimental (circles) two-photon excitation spectra of molecules 1 (a), 2 (b), and $\mathbf{3}$ (c) in toluene. Dashed lines refer to TD-B3LYP diagonal contributions, continuous lines to the whole TD-B3LYP response. Dotted lines: excitonic results.

framework of the Frenkel exciton model. For these results, dipole moment values obtained with quantum-chemical calculations for the monomeric model have been used for each dipolar branch; the value of $0.14 \mathrm{eV}$ has been exploited for the excitonic coupling (which is consistent with a point-dipole distance of $8 \AA$ ); an angle of $120^{\circ}$ has been imposed between the dipolar units. No local-field factor corrections have been introduced. While the qualitative agreement of these results with experimental spectra is reasonable, one can notice that the enhancement of the TPA cross-section with the number of branches is caught but underestimated by the excitonic calculation (see also Supporting Information).

The same figure reports TPA spectra obtained by ab initio calculation. For a better comparison, calculated spectra have been obtained by introducing a local-field factor (spherical cavity), which accounts for toluene $(n=1.494)$ as the solvent. Given the uncertainty of the TPA cross-section line width, we did not apply more sophisticated cavity/solvent models. However, to emphasize the expected trends, we conducted TDB3LYP calculations of the excited structure and TPA profile using the polarizable continuum model implemented in Gaussian $98 .{ }^{64}$ In all molecules in question, these calculations show a consistent solvatochromic red-shift of TPA excitation frequen- 
cies by $\sim 30-60 \mathrm{meV}$ and an increase of the corresponding cross-sections by $\sim 10 \%$ (most notably in the dipolar compound). In Figure 12, responses calculated by taking into account the diagonal contributions only (dashed lines) and the total results (diagonal + nondiagonal) (continuous lines) are reported. Obviously, only diagonal terms contribute to the TPA response of the dipolar chromophore $\mathbf{1}$ (panel a), while for compounds $\mathbf{2}$ and $\mathbf{3}$, off-diagonal contributions are nonnegligible. In particular, for chromophore 2 (panel b), one can notice that the lowestenergy band (corresponding to the most intense OPA) is twophoton-allowed only through off-diagonal tensor components, as can be predicted by symmetry. The second (higher-energy) band is instead dominated by diagonal terms, even though nondiagonal contributions are apparent. For chromophore $\mathbf{3}$ (panel c), both bands are two-photon-allowed through diagonal terms, but they both have large off-diagonal contributions. The global agreement between experimental and calculated spectra is good: apart from deviations due to global line width, computational results for chromophores $\mathbf{1}$ and $\mathbf{2}$ are very satisfactory. The comparison formally worsens for the higherenergy band of chromophore $\mathbf{3}$, which experimentally is displaced toward higher energy (the error is within $0.1 \mathrm{eV}$ and still provides a fortuitous consistency between theory and experiment). Calculations predict a strong enhancement due to the two degenerate excited states, with a maximum at about $730 \mathrm{~nm}$. However, the experimental TPA cross-section still increases between 730 and $705 \mathrm{~nm}$. This might be due to a higher-lying intense TPA-allowed excited state in the red-visible region. This state should be related to the second excited state of the dipolar branches, which shows up at $\lambda_{\mathrm{abs}}=300 \mathrm{~nm}$. As a matter of fact, a more detailed analysis is prevented by the limited experimental data in the red-visible region. The branching enhancement in the long-wavelength region is also underestimated by calculations: this can be explained by the fact that calculations disregard molecular vibrations and inhomogeneous broadening effects (due, for example, to conformational disorder for branched chromophores, which increases with an increasing number of branches).

The large, amplified TPA response shown by branched chromophores is related to the interaction between the branched dipolar unities. We stress that this enhancement is underestimated by the Frenkel exciton approach, while it is reproduced much better by quantum-chemical calculations using the supramolecular approach (see Figure 12 and Supporting Information). Thus, the correct prediction of the enhancement in the TPA response of branched chromophores requires accounting for the presence of coherent interactions between branches (beyond dipolar model) ${ }^{107}$ and higher-lying excited states. ${ }^{27}$ Moreover, we observe that the three-branched compound leads to a larger cooperative effect than the analogous two-branched systems. This suggests that further TPA enhancement could be achieved in $n$-branched systems $(n>3)$ built from a core allowing significant coupling between the branches and from branches ensuring pronounced intramolecular charge transfer between the center and the periphery upon excitation.

\section{Conclusion}

A deeper understanding of the branching effect of dipolar entities on linear and nonlinear optical properties of multichromophoric structures has been achieved by a combined theoretical and experimental approach on a series of branched structures made up with prototype chromophores.

Theoretical modeling of one- and two-photon absorption spectra using time-dependent density functional theory is in very good agreement with experiment. In particular, an agreement within $0.1-0.2 \mathrm{eV}$ for excited-state energies (for both absorption and emission spectra) and 50\% for absolute amplitudes of TPA cross-sections (given the uncertainty of line widths and solvent effects) is achieved. Such a good performance of the TD-DFT approach can be partially rationalized by a "single-particle" nature of all excited states involved. HF (TD-HF) geometries of ground (excited) states and the B3LYP functional for excitedstate calculations proved to be a successful blend of quantumchemical approaches for the given molecular systems. Complementary analyses of calculated spectroscopic observables and natural transition orbitals further allowed us to confirm experimental trends and understand the underlying physical phenomena.

Both experimental and theoretical findings consistently show that, while the absorbing state of the branched molecules can be qualitatively understood in terms of delocalized Frenkel exciton states, the emitting state is localized on a single branch, corresponding to a breakage of coherent coupling between the arms. Our calculations demonstrate that this localization is a consequence of nuclear relaxation, suggesting that this phenomenon has quite a general appearance in multibranched structures and is not linked to particular environmental conditions, nor to the peculiar chromophores studied in this paper. The predicted and observed localized nature of the emitting state allows the maintainance of high fluorescence quantum yield, a very important feature for imaging applications.

The interaction between branched chromophores is also responsible for splitting between degenerate excited states, so that the amount of coupling can be extracted within the Frenkel exciton scheme. The value thus extracted agrees well with the calculated estimate and can also be assessed through a pointlike dipole model. A simple excitonic splitting is already responsible for TPA enhancement in regions where TPA bands of the different (branched and nonbranched) compounds overlap and for TPA activation in spectral regions where the dipolar analogue is almost two-photon transparent. However, we clearly demonstrate (through the comparison of excitonic results with ab initio calculations) that the presence of coherent interactions between branches and high-energy excited states provides a significant TPA enhancement as well. It should be stressed that the enhancement is much larger than experimental errors and that the femtosecond regime excludes any contribution from excited-state absorption and artificial enhancement. It is also worthwhile to stress that single-wavelength measurements of TPA cross-sections are not enough to estimate the possible enhancement of the response: a large spectral region should be investigated because of Davydov's splittings due to excitonic interactions. Also, close relations between one- and two-photon absorption spectra exist due to symmetry, even if they are not always recognized. These allow simple estimates of TPA maxima starting from OPA data. This, together with the use of simple approaches, such as the Frenkel exciton model, can constitute a powerful guideline for the rational design of multichromophoric structures with optimized response, by taking advantage from symmetry, electronic coupling, and coherent interactions.

We argue that the appropriate tuning of the number of branches, the coupling between them, the symmetry, and the modulation of the intramolecular charge transfer from the core to the periphery could constitute a substantial way for obtaining amplification of the aimed properties in the desired spectral regions. This is not only appealing for various applications, but it also represents an opportunity on a more fundamental vein. 
Here, we demonstrate that a more rational design of optimized structures can be achieved through a "bottom-up" approach that involves not only the synthetic route (from molecular to supramolecular) but also the interpretative and modeling approaches.

Acknowledgment. M.B.D. acknowledges partial financial support from CNRS (NOI grant) and Rennes Métropole. L.P. received a fellowship from the French Ministère de l'Education Nationale, de l'Enseignement Supérieur, et de la Recherche. F.T. acknowledges support by a Marie Curie Intra-European Fellowship within the Sixth European Community Framework Program. Part of the calculations were supported by the "Centre Informatique National de l'Enseignement Supérieur" (CINESFrance). We wish to thank Dr. Bharath Kumar Goud Bhatthula for his contribution to the synthesis and Dr. Artem Masunov for stimulating discussions. The research at LANL is supported by the Center for Nonlinear Studies (CNLS) and the LDRD program of the U.S. Department of Energy. This support is gratefully acknowledged.

Supporting Information Available: Four figures: Figure 1 shows the deconvolution of the normalized absorption spectra of chromophore 2 using the one of chromophore 1. Figures 2 and 3 show the solvatochromic behavior of chromophores 1 and 2, respectively. Figure 4 reports normalized experimental and calculated TPA spectra. This material is available free of charge via the Internet at http://pubs.acs.org.

\section{References and Notes} 103.

(1) Marder, S. R.; Beratan, D. N.; Cheng, L. T. Science 1991, 252,

(2) Kanis, D. R.; Ratner, M. A.; Marks, T. J. Chem. Rev. 1994, 94, 195.

(3) Brédas, J.-L.; Cornil, K.; Meyers, F.; Beljonne, D. In Handbook of Conducting Polymers; Skotheim, T. A., Elsenbaumer, R. L., Reynolds, J. R., Eds.; Marcel Dekker: New York, 1998; p 1.

(4) He, G. S.; Xu, G. C.; Prasad, P. N.; Reinhardt, B. A.; Bhatt, J. C.; McKellar, R.; Dillard, A. G. Opt. Lett. 1995, 20, 435.

(5) Ehrlich, J. E.; Wu, X. L.; Lee, I.-Y. S.; Hu, Z.-Y.; Röckel, H.; Marder, S. R.; Perry, J. W. Opt. Lett. 1997, 22, 1843.

(6) Reinhardt, B. A.; Brott, L. L.; Clarson, S. J.; Dillard, A. G.; Bhatt, J. C.; Kannan, R.; Yuan, L.; He, G. S.; Prasad, P. N. Chem. Mater. 1998, $10,1863$.

(7) Albota, M.; Beljonne, D.; Brédas, J.-L.; Ehrlich, J. E.; Fu, J.-Y.; Heikal, A. A.; Hess, S. E.; Kogej, T.; Levin, M. D.; Marder, S. R.; McCordMaughon, D.; Perry, J. W.; Röckel, H.; Rumi, M.; Subramaniam, G.; Webb, W. W.; Wu, X.-L.; Xu, C. Science 1998, 281, 1653.

(8) Ventelon, L.; Blanchard-Desce, M.; Moreaux, L.; Mertz, J. Chem. Cотmun. 1999, 2055.

(9) Kim, O.-K.; Lee, K.-S.; Woo, H. Y.; Kim, K.-S.; He, G. S.; Guang, S. H.; Swiatkiewicz, J.; Prasad, P. N. Chem. Mater. 2000, 12, 284.

(10) Ventelon, L.; Charier, S.; Moreaux, L.; Mertz, J.; Blanchard-Desce, M. Angew. Chem., Int. Ed. 2001, 40, 2098.

(11) Frederiksen, P. K.; Jørgensen, M.; Ogilby, P. R. J. Am. Chem. Soc. 2001, 123, 1215.

(12) Ventelon, L.; Moreaux, L.; Mertz, J.; Blanchard-Desce, M. Synth. Met. 2002, 127, 17.

(13) Mongin, O.; Porrès, L.; Moreaux, L.; Mertz, J.; Blanchard-Desce, M. Org. Lett. 2002, 4, 719.

(14) Pond, S. J. K.; Rumi, M.; Levin, M. D.; Parker, T. C.; Beljonne, D.; Day, M. W.; Brédas, J.-L.; Marder, S. R.; Perry, J. W. J. Phys. Chem. A 2002, 106, 11470.

(15) Abbotto, A.; Beverina, L.; Bozio, R.; Facchetti, A.; Ferrante, C.; Pagani, G. A.; Pedron, D.; Signorini, R. Org. Lett. 2002, 4, 1495.

(16) Strehmel, B.; Sarker, A. M.; Detert, H. ChemPhysChem 2003, 4 249.

(17) Kim, O. K.; Lee, K. S.; Huang, Z.; Heuer, W. B.; Paik-Sung, C. S. Opt. Mater. 2003, 21, 559.

(18) Yang, W. J.; Kim, D. Y.; Jeong, M.-Y.; Kim, H. M.; Jeon, S.-J.; Cho, B. R. Chem. Commun. 2003, 2618.

(19) Iwase, Y.; Kamada, K.; Ohta, K.; Kondo, K. J. Mater. Chem. 2003, 13,1575 .
(20) Kawamata, J.; Akiba, M.; Tani, T.; Harada, A.; Inagaki, Y. Chem. Lett. 2004, 33, 448 .

(21) Cho, B. R.; Lee, S. J.; Lee, S. H.; Son, K. H.; Kim, Y. H.; Doo, J.-Y.; Lee, G. J.; Kang, T. I.; Lee, Y. K.; Cho, M.; Jeon, S.-J. Chem. Mater. 2001, 13, 1438

(22) Zyss, J.; Ledoux, I. Chem. Rev. 1994, 94, 77.

(23) Joshi, M. P.; Swiatkiewicz, J.; Xu, F.; Prasad, P. N.; Reinhardt, B. A.; Kannan, R. Opt. Lett. 1998, 23, 1742.

(24) Chung, S.-J.; Kim, K.-S.; Lin, T.-C.; He, G. S.; Swiatkiewicz, J.; Prasad, P. N. J. Phys. Chem. B 1999, 103, 10741.

(25) He, G. S.; Swiatkiewicz, J.; Jiang, Y.; Prasad, P. N.; Reinhardt, B. A.; Tan, L.-S.; Kannan, R. J. Phys. Chem. A 2000, 104, 4805.

(26) Cho, B. R.; Son, K. H.; Sang, H. L.; Song, Y.-S.; Lee, Y.-K.; Jeon, S.-J.; Choi, J. H.; Lee, H.; Cho, M. J. Am. Chem. Soc. 2001, 123, 10039.

(27) Beljonne, D.; Wenseleers, W.; Zojer, E.; Shuai, Z.; Vogel, H.; Pond, S. J. K.; Perry, J. W.; Marder, S. R.; Brédas, J.-L. Adv. Funct. Mater. 2002, 12,631 .

(28) Mongin, O.; Brunel, J.; Porrès, L.; Blanchard-Desce, M. Tetrahedron Lett. 2003, 44, 2813.

(29) Mongin, O.; Porrès, L.; Katan, C.; Pons, T.; Mertz, J.; BlanchardDesce, M. Tetrahedron Lett. 2003, 44, 8121.

(30) Porrès, L.; Mongin, O.; Katan, C.; Charlot, M.; Pons, T.; Mertz, J.; Blanchard-Desce, M. Org. Lett. 2004, 6, 47.

(31) Lee, H. J.; Sohn, J.; Hwang, J.; Park, S. Y.; Choi, H.; Cha, M. Chem. Mater. 2004, 16, 456.

(32) Yang, W. J.; Kim, D. Y.; Kim, C. H.; Jeong, M.-Y.; Lee, S. K. Jeon, S.-J.; Cho, B. R. Org. Lett. 2004, 6, 1389.

(33) Meng, F.; Li, B.; Qian, S.; Chen, K.; Tian, H. Chem. Lett. 2004, 33,470 .

(34) Spangler, C. W.; Elandaloussi, E. H.; Casstevens, M. K.; Kumar, D. N.; Weibel, J. F.; Burzynski, R. Proc. SPIE-Int. Soc. Opt. Eng. 1999, 3798,117

(35) McDonagh, A. M.; Humphrey, M. G.; Samoc, M.; Luther-Davies, B. Organometallics 1999, 18, 5195.

(36) Adronov, A.; Fréchet, J. M. J.; He, G. S.; Kim, K.-S.; Chung, S.J.; Swiatkiewicz, J.; Prasad, P. N. Chem. Mater. 2000, 12, 2838.

(37) Chung, S.-J.; Lin, T.-C.; Kim, K.-S.; He, G. S.; Swiatkiewicz, J.; Prasad, P. N.; Baker, G. A.; Bright, F. V. Chem. Mater. 2001, 13, 4071. (38) Drobizhev, M.; Karotki, A.; Rebane, A.; Spangler, C. W. Opt. Lett. 2001, 26, 1081.

(39) Drobizhev, M.; Karotki, A.; Kruk, M.; Mamardashvili, N. Z.; Rebane, A. Chem. Phys. Lett. 2002, 361, 504.

(40) Abbotto, A.; Beverina, L.; Bozio, R.; Facchetti, A.; Ferrante, C.; Pagani, G. A.; Pedron, D.; Signorini, R. Chem. Commun. 2003, 2144.

(41) Drobizhev, M.; Karotki, A.; Dzenis, Y.; Rebane, A.; Suo, Z.; Spangler, C. W. J. Phys. Chem. B 2003, 107, 7540.

(42) Yoo, J.; Yang, S. K.; Jeong, M.-Y.; Ahn, H. C.; Jeon, S.-J.; Cho, B. R. Org. Lett. 2003, 5, 645.

(43) Liu, S.; Lin, K. S.; Churikov, V. M.; Su, Y. Z.; Lin, J. T. s.; Huang, T.-H.; Hsu, C. C. Chem. Phys. Lett. 2004, 390, 433.

(44) Goodson, T. G., III Acc. Chem. Res. 2005, 38, 99.

(45) Denk, W.; Strickler, J. H.; Webb, W. W. Science 1990, 248, 73.

(46) Xu, C.; Zipfel, W.; Shear, J. B.; Williams, R. M.; Webb, W. W. Proc. Natl. Acad. Sci. U.S.A. 1996, 93, 10763.

(47) Larson, D. R.; Zipfel, W. R.; Williams, R. M.; Clark, S. W.; Bruchez, M. P.; Wise, F. W.; Webb, W. W. Science 2003, 300, 1434.

(48) Bhawalkar, J. D.; Kumar, N. D.; Zhao, C. F.; Prasad, P. N. J. Clin. Laser Med. Surg. 1997, 15, 201.

(49) Maruo, S.; Nakamura, O.; Kawata, S. Opt. Lett. 1997, 22, 132.

(50) Cumpston, B. H.; Ananthavel, S. P.; Barlow, S.; Dyer, D. L.; Ehrlich, J. E.; Erskine, L. L.; Heikal, A. A.; Kuebler, S. M.; Lee, I.-Y. S.; McCord-Maughon, D.; Qin, J.; Röckel, H.; Rumi, M.; Wu, X. L.; Marder, S. R.; Perry, J. W. Nature (London) 1999, 398, 51.

(51) Kawata, S.; Sun, H.-B.; Tanaka, T.; Takada, K. Nature (London) 2001, 412, 697 .

(52) Zhou, W.; Kuebler, S. M.; Braun, K. L.; Yu, T.; Cammack, J. K.; Ober, C. K.; Perry, J. W.; Marder, S. R. Science 2002, 296, 1106.

(53) Parthenopoulos, D. A.; Rentzepis, P. M. Science 1989, 245, 843.

(54) Strickler, J. H.; Webb, W. W. Opt. Lett. 1991, 16, 1780.

(55) Dvornikov, A. S.; Rentzepis, P. M. Opt. Commun. 1995, 119, 341

(56) Belfield, K. D.; Schafer, K. J. Chem. Mater. 2002, 14, 3656.

(57) Lin, T. C.; Chung, S. J.; Kim, K. S.; Wang, X. P.; He, G. S.; Swiatkiewicz, J.; Pudavar, H. E.; Prasad, P. N. In Polymers for Photonics Applications II; Lee, K.-S., Ed.; Springer-Verlag GmbH: Berlin, 2003; Vol. 161, p 157.

(58) Stadler, S.; Feiner, F.; Bräuchle, C.; Brandl, S.; Gompper, R. Chem. Phys. Lett. 1995, 245, 292.

(59) Stadler, S.; Bräuchle, C.; Brandl, S.; Gompper, R. Chem. Mater. 1996, 8, 414.

(60) Lambert, C.; Gaschler, W.; Schmälzlin, E.; Meerholz, K.; Bräuchle, C. J. Chem. Soc., Perkin Trans. 2 1999, 2, 577.

(61) Lambert, C.; Gaschler, W.; Noll, G.; Weber, M.; Schmalzlin, E.; Brauchle, C.; Meerholz, K. J. Chem. Soc., Perkin Trans. 2 2001, 964. 
(62) Lai, G.; Bu, X. R.; Santos, J.; Mintz, E. A. Synlett 1997, 11, 1275.

(63) Elandaloussi, E. H.; Spangler, C. W. Polym. Prepr. (Am. Chem. Soc., Div. Polym. Chem.) 1998, 39, 1055.

(64) Frisch, M. J.; Trucks, G. W.; Schlegel, H. B.; Scuseria, G. E.; Robb,

M. A.; Cheeseman, J. R.; Zakrzewski, V. G.; Montgomery, J. A., Jr.; Stratmann, R. E.; Burant, J. C.; Dapprich, S.; Millam, J. M.; Daniels, A. D.; Kudin, K. N.; Strain, M. C.; Farkas, O.; Tomasi, J.; Barone, V.; Cossi, M.; Cammi, R.; Mennucci, B.; Pomelli, C.; Adamo, C.; Clifford, S.; Ochterski, J.; Petersson, G. A.; Ayala, P. Y.; Cui, Q.; Morokuma, K.; Malick D. K.; Rabuck, A. D.; Raghavachari, K.; Foresman, J. B.; Cioslowski, J.; Ortiz, J. V.; Stefanov, B. B.; Liu, G.; Liashenko, A.; Piskorz, P.; Komaromi, I.; Gomperts, R.; Martin, R. L.; Fox, D. J.; Keith, T.; Al-Laham, M. A.; Peng, C. Y.; Nanayakkara, A.; Gonzalez, C.; Challacombe, M.; Gill, P. M. W.; Johnson, B. G.; Chen, W.; Wong, M. W.; Andres, J. L.; Head-Gordon, M.; Replogle, E. S.; Pople, J. A. Gaussian 98, revision A.11; Gaussian, Inc.: Pittsburgh, PA, 1998.

(65) Masunov, A.; Tretiak, S. J. Phys. Chem. B 2004, 108, 899.

(66) Furche, F.; Ahlrichs, R. J. Chem. Phys. 2002, 117, 7433.

(67) Della Sala, F.; Görling, A. J. Chem. Phys. 2001, 115, 5718.

(68) Casida, M. E.; Gutierrez, F.; Guan, J.; Gadea, F.-X.; Salahub, D.; Daudey, J.-P. J. Chem. Phys. 2000, 113, 7062.

(69) Tozer, D. J. J. Chem. Phys. 2003, 119, 12697

(70) Casida, M. E.; Jamorski, C.; Casida, K. C.; Salahub, D. R. J. Chem. Phys. 1998, 108, 4439.

(71) Onida, G.; Reining, L.; Rubio, A. Rev. Mod. Phys. 2002, 74, 601.

(72) Tretiak, S.; Chernyak, V. J. Chem. Phys. 2003, 119, 8809.

(73) Bartholomew, G. P.; Rumi, M.; Pond, S. J.; Perry, J. W.; Tretiak, S.; Bazan, G. C. J. Am. Chem. Soc. 2004, 126, 11529. 444 .

(74) Kobko, N.; Masunov, A.; Tretiak, S. Chem. Phys. Lett. 2004, 392,

(75) Garito, A. F.; Heflin, J. R.; Wong, K. Y.; Zamani-Khamiri, O. In Organic Materials for Non-linear Optics; Hann, R. A., Bloor, D., Eds.; Royal Society of Chemistry: London, 1989; p 16.

(76) Ahlrichs, R.; Bär, M.; Häser, M.; Horn, H.; Kölmel, C. Chem. Phys. Lett. 1989, 162, 165.

(77) McQuarrie, D. A.; Simon, J. D. Physical chemistry. A molecular approach; University Science Books: Sausalito, CA, 1997.

(78) Martin, R. L. J. Chem. Phys. 2003, 118, 4775.

(79) Franco, I.; Tretiak, S. J. Am. Chem. Soc. 2004, 126, 12130

(80) Kokalj, A. J. Mol. Graphics Mod. 1999, 17, 176. Code available from http://www.xcrysden.org/.

(81) Davidov, A. S. Theory of molecular excitons; Plenum Press: New York, 1971.

(82) Silinsh, E. A.; Capek, V. Organic Molecular Crystals: Interaction, Localization, and Transport Phenomena; AIP Press: New York, 1994.

(83) Spano, F. C.; Knoester, J. In Advances in Magnetic and Optical Resonance; Waren, W. S., Ed.; Academic Press: New York, 1994; Vol. $18, \mathrm{p} 117$.

(84) J-Aggregates; Kobayashi, T., Ed.; World Scientific: Singapore, 1996.

(85) Hu, X.; Damjanovic, A.; Ritz, T.; Schulten, K. Proc. Natl. Acad. Sci. U.S.A. 1998, 95, 5935
(86) Sundström, V.; Pullerits, T.; Van Grondelle, R. J. Phys. Chem. B 1999, 103, 2327

(87) Tretiak, S.; Chernyak, V.; Mukamel, S. J. Phys. Chem. B 1998, $102,3310$.

(88) Orr, B. J.; Ward, J. F. Mol. Phys. 1971, 20, 513.

(89) Demas, J. N.; Crosby, G. A. J. Phys. Chem. 1971, 75, 991

(90) Xu, C.; Webb, W. W. J. Opt. Soc. Am. B 1996, 13, 481.

(91) Albota, M. A.; Xu, C.; Webb, W. W. Appl. Opt. 1998, 37, 7352.

(92) Lippert, E. Z. Naturforsch., A 1955, 10, 541.

(93) Mataga, N.; Kaifu, Y.; Koizumi, M. Bull. Chem. Soc. Jpn. 1955 28,690 .

(94) Sobolev, A. N.; Belsky, V. K.; Romm, I. P.; Chernikova, N. Y.; Guryanova, E. N. Acta Crystallogr., Sect. C: Cryst. Struct. Commun. 1985, C41, 967.

(95) Brédas, J.-L.; Cornil, J.; Beljonne, D.; Dos Santos, D. A.; Shuai, Z. Acc. Chem. Res. 1999, 32, 267.

(96) Tretiak, S.; Saxena, A.; Martin, R. L.; Bishop, A. R. Phys. Rev. Lett. 2002, 89, 097402.

(97) Jamorski Jodicke, C.; Luthi, H. P. J. Am. Chem. Soc. 2003, 125,

(98) Rappoport, D.; Furche, F. J. Am. Chem. Soc. 2004, 126, 1277.

(99) Molecular Nonlinear Optics: Materials, Physics, and Devices; Zyss, J., Ed.; Academic Press: Boston, 1993.

(100) Moran, A. M.; Myers Kelley, A.; Tretiak, S. Chem. Phys. Lett. 2002, 367, 293.

(101) Suppan, P. J. Photochem. Photobiol., A 1990, 50, 293.

(102) Strickler, S. J.; Berg, R. A. J. Chem. Phys. 1962, 37, 814.

(103) This difference is mainly attributed to the approximate nature of eq 4, which lumps all oscillator strengths into a single electronic transition and does not discriminate between separate electron-vibrational transitions (i.e., vibrational effects/conformational disorder and solvent/thermal bath may only reduce radiative lifetime compared to the ideal case). Larger calculated "emitting" transition dipoles contribute to the observed discrepancy as well. In fact, eq 4 shows that an estimate of the transition dipole moment too large by a factor of the square root of 2 will lead to a twice smaller lifetime.

(104) Fidder, H.; Knoester, J.; Wiersma, D. A. J. Chem. Phys. 1991, 95,7880 .

(105) Verbouwe, W.; Viaene, L.; Van der Auweraer, M.; De Schryver, F. C.; Masuhara, H.; Pansu, R.; Faure, J. J. Phys. Chem. A 1997, 101, 8157 (106) Verbouwe, W.; Van der Auweraer, M.; De Schryver, F. C.; Piet, J. J.; Warman, J. M. J. Am. Chem. Soc. 1998, 120, 1319.

(107) Lahankar, S. A.; West, R.; Varnavski, O.; Xie, X.; Goodson, T. III J. Chem. Phys. 2004, 120, 337.

(108) Varnavski, O. P.; Ostrowski, J. C.; Sukhomlinova, L.; Twieg, R. J.; Bazan, G., C.; Goodson, T., III J. Am. Chem. Soc. 2002, 124, 1736.

(109) Thompson, A. L.; Gaab, K. M.; Xu, J.; Bardeen, C. J.; Martínez, T. J. J. Phys. Chem. A 2004, 108, 671.

(110) Gaab, K. M.; Thompson, A. L.; Xu, J.; Martínez, T. J.; Bardeen, C. J. J. Am. Chem. Soc. 2003, 125, 9288.

(111) Kuzyk, M. G. J. Chem. Phys. 2003, 119, 8327. 